Research papers

\title{
An overview on the use of backscattered sound for measuring suspended particle size and concentration profiles in non-cohesive inorganic sediment transport studies
}

\author{
Peter D. Thorne ${ }^{\mathrm{a}, *}$, David Hurther ${ }^{\mathrm{b}}$ \\ a National Oceanography Centre, Joseph Proudman Building, 6 Brownlow Street, Liverpool L3 5DA, Merseyside, United Kingdom \\ ${ }^{\mathrm{b}}$ Laboratory of Geophysical and Industrial Flows (LEGI), CNRS UMR 5519, Grenoble University, France
}

\section{A R T I C L E I N F O}

\section{Article history:}

Received 20 June 2013

Received in revised form

18 October 2013

Accepted 24 October 2013

Available online 4 November 2013

Keywords:

Overview analysis

Sandy suspended sediments

Size and concentration profiles

Multi-frequency acoustic backscattering

Inversion methodologies

Sediment transport processes

\begin{abstract}
A B S T R A C T
For over two decades, coastal marine scientists studying boundary layer sediment transport processes have been using, and developing, the application of sound for high temporal-spatial resolution measurements of suspended particle size and concentration profiles. To extract the suspended sediment parameters from the acoustic data requires an understanding of the interaction of sound with a suspension of sediments and an inversion methodology. This understanding is distributed around journals in a number of scientific fields and there is no single article that succinctly draws together the different components. In the present work the aim is to provide an overview on the acoustic approach to measuring suspended sediment parameters and assess its application in the study of non-cohesive inorganic suspended sediment transport processes.
\end{abstract}

Crown Copyright @ 2013 Published by Elsevier Ltd. All rights reserved.

\section{Introduction}

It is readily acknowledged that one of the most important processes in the coastal environment is the movement of sediments; they impact on habitats, water quality, turbidity, biogeochemistry and morphology (Davies and Thorne, 2008; Amoudry and Souza, 2011). Therefore improving our capability to monitor and model sediment transport in the marine environment is an essential component of sustainable development and management. In particular as detailed physics based process models have developed, there has been the requirement to measure sediment dynamics with increasing temporal-spatial resolution (van der Werf et al., 2008). This has led to the development of new technologies and the utilisation of acoustics has been one of the competitors in this field (Thorne and Hay, 2012).

On the larger scale acoustic Doppler current profilers, ADCP, have been around for three decades measuring flow profiles (Gordon, 1996) and more recently the amplitude of the backscattered signal has been used to estimate suspended concentration (Holdaway et al., 1999; Moore et al., 2012, 2013). Further in the last year or two there has also been the application of using swath bathymetry systems to measure suspended sediments

\footnotetext{
*Corresponding author. Tel.: +44 151795 4862; fax: +44151795 4801.

E-mail address: pdt@noc.ac.uk (P.D. Thorne).
}

(Simmons et al., 2010). On the smaller scale significant advances have been taking place in the application of sound to the study of near-bed sediment transport processes (Vincent et al., 1999; Hurther and Thorne, 2011; Bolanos et al., 2012; Hay et al., 2012). Acoustics has and is being developed for near-bed studies because it is recognised as having the potential to measure non-intrusively, co-located, simultaneously and with high spatial-temporal resolution, suspended sediment and flow profiles and provide information on bedforms. This has lead to the development of multi-frequency acoustic backscatter systems, ABS, to measure suspended particle size and concentration (Crawford and Hay, 1993; Thorne and Hardcastle, 1997), high resolution acoustic Doppler velocity profilers, ADVP, for turbulent and intra-wave observations (Hurther et al., 2007; Hay et al., 2012), combined systems for high resolution acoustic concentration and velocity profiles, ACVP, (Hurther et al., 2011) and two and three dimensional acoustic ripple profiles, ARP, to provide detailed bedform measurements with sub-centimetric resolution (Traykovski, 2007; Hay, 2011; O'Hara Murray et al., 2012). Developments using acoustic near-bed systems have been presented in the literature (Thorne and Hanes, 2002; Hurther et al., 2011).

The present work focuses on the near-bed application and forms the third part of a trilogy on ABS systems in which the first part described the system calibration using suspensions with known scattering characteristics (Betteridge et al., 2008), the second centered on acoustic scattering properties of suspended 


\begin{tabular}{|c|c|c|c|}
\hline \multicolumn{2}{|c|}{ Nomenclature } & \multirow{2}{*}{$\begin{array}{l}V_{m}^{2} \\
x=k a \\
z\end{array}$} & \multirow{2}{*}{$\begin{array}{l}\text { mean-square backscattered signal }\left(\mathrm{V}^{2}\right) \\
(-), x_{o}=k a_{\mathrm{o}}(-) \\
\text { height above the bed }(\mathrm{m})\end{array}$} \\
\hline$a$ & particle radius $(\mathrm{m})$ & & \\
\hline$a_{o}$ & mean radius based on $n(a)(\mathrm{m})$ & $z_{r}$ & reference height, $z_{r}=0.005(\mathrm{~m})$ \\
\hline$a_{c}$ & mean radius of the suspension field based on $n(a)(m)$ & $\alpha_{w}$ & attenuation due to water absorption (Nepers $\mathrm{m}^{-1}$ ) \\
\hline$a_{m}$ & mean radius based on $m(a)(\mathrm{m})$ & $\alpha_{s}$ & attenuation due to sediment scattering (Nepers $\mathrm{m}^{-1}$ ) \\
\hline$a_{M}$ & acoustic estimate of the suspended particle radius (m) & $\gamma_{o}$ & random error introduced into the backscattered \\
\hline$a_{r}$ & reference value for $a_{c}$ at $z_{r}(\mathrm{~m})$ & & signal (-) \\
\hline$a_{50}$ & median mass radius based on $m(a)(\mathrm{m})$ & $\delta$ & normalised standard deviation (-) \\
\hline$A_{t}$ & transducer radius $(\mathrm{m})$ & $\varepsilon$ & systematic error introduced into the backscattered \\
\hline C & suspended concentration field $\left(\mathrm{kg} \mathrm{m}^{-3}\right)$ & & signal (-) \\
\hline$C_{r}$ & reference value for $C$ at $z_{r}\left(\mathrm{~kg} \mathrm{~m}^{-3}\right)$ & $\eta$ & number of independent samples (-) \\
\hline$d_{50}$ & median mass diameter based on $m(a)(\mathrm{m})$ & $\dot{\lambda}$ & wavelength of sound (m) \\
\hline$f_{i}$ & intrinsic backscatter form function (-) & $\xi$ & sediment attenuation constant $\left(\mathrm{kg}^{-1} \mathrm{~m}^{2}\right)$ \\
\hline$f$ & $\begin{array}{l}\text { ensemble density normalised backscatter form func- } \\
\text { tion }\left(\mathrm{kg}^{-1 / 2} \mathrm{~m}^{3 / 2}\right)\end{array}$ & $\begin{array}{l}\rho \\
\sigma\end{array}$ & $\begin{array}{l}\text { sediment grain density }\left(\mathrm{kg} \mathrm{m}^{-3}\right) \\
\text { standard deviation (units depend on parameter) }\end{array}$ \\
\hline$j$ & frequency counter used in the inversion. & $\sigma_{e}$ & standard error (units depend on parameter) \\
\hline$k$ & acoustic wavenumber, $2 \pi / \lambda\left(\mathrm{m}^{-1}\right)$ & $\tau$ & time lag $(\mathrm{s})$ \\
\hline$K$ & sediment backscattering property $\left(\mathrm{kg}^{-1 / 2} \mathrm{~m}\right)$ & $\tau_{o}$ & decorrelation time lag (s) \\
\hline$m(\mathrm{a})$ & particle mass radius probability density function (-) & $\Phi$ & parameter used to estimate acoustical particle \\
\hline$M_{o}$ & initial acoustic estimate of the suspended concentra- & & radius $(-)$ \\
\hline & tion $\left(\mathrm{kg} \mathrm{m}^{-3}\right)$ & $\chi_{i}$ & intrinsic normalised total scattering cross-section (-) \\
\hline$M$ & $\begin{array}{l}\text { acoustic estimate of the suspended concentration. } \\
\left(\mathrm{kg} \mathrm{m}^{-3}\right)\end{array}$ & $\chi$ & $\begin{array}{l}\text { ensemble density normalised mean normalised total } \\
\text { scattering cross-section }\left(\mathrm{kg}^{-1} \mathrm{~m}^{3}\right)\end{array}$ \\
\hline$n(\mathrm{a})$ & particle number radius probability density function (-) & $\psi$ & Transducer nearfield correction (-) \\
\hline$N$ & number of frequencies used in the inversion. & 1 & parameters in the text with subscript ' $b$ ' refers to bed \\
\hline$r$ & range from the transducer $(\mathrm{m})$ & & sediments. \\
\hline$r_{n}$ & transducer nearfield $\pi A_{t}^{2} / \lambda(\mathrm{m})$ & 2 & $\bar{X}$. Overbar represents a time averaged parameter. \\
\hline$\Re$ & the system constant $\left(\mathrm{V} \mathrm{m}^{3 / 2}\right)$ & 3 & $\bar{X} / Y$. Overbar represents a time and height averaged \\
\hline V & backscattered signal (V) & & parameter. \\
\hline$u^{*}$ & bed friction velocity $\left(\mathrm{ms}^{-1}\right)$ & & \\
\hline
\end{tabular}

marine sediments (Thorne and Meral, 2008; Moate and Thorne, 2012) and here the study focuses upon the extraction of suspended sediment parameters from the backscattered acoustic data. The literature associated with the application of acoustics to the measurement of suspended sediment measurements is distributed amongst journals in the fields of acoustics (Sheng and Hay, 1988; Thosteson and Hanes, 1998; Moate and Thorne, 2009), engineering (Thorne et al., 1994; Hurther et al., 2011), sedimentology (Vincent et al., 1991, 1999; O’Hara Murray et al., 2012; Pedocchi and Garcia, 2012) and geosciences (Hay and Sheng, 1992; Hurther and Thorne, 2011) with no single article providing an overview of the approach. It was therefore considered timely, given the expanding use of sound for measuring suspended sediments, that an article providing such an overview of the topic would be of value to coastal marine scientists who are using, or beginning to use acoustics, in bottom boundary layer sediment transport studies.

Examined here is the inversion of acoustic data backscattered from inorganic non-cohesive suspended sediments and its application to the measurement of suspended particle size and concentration profiles. The intention is to provide a description of the commonly adopted inversion approach used with contemporary ABS deployed in sandy marine environments. To this end a series of formulations are described which cover a range of inversions from simple to more complex approaches. Depending on the availability of independent information on the suspension, the inversion may be more or less subject to uncertainty. Here an assessment is made of the inversion process and how different factors impact on the calculated acoustic estimates of suspended particle size and concentration. The relationship between the measured acoustic parameters and those commonly used in sediment transport models is discussed. The influence of the system calibration accuracy, the uncertainty in the scattering properties of the sediments, the effect of the particle size distribution, the frequencies to use and the impact of signal averaging are all considered in the assessment. The specific impact of extraneous scatterers such as bubbles, organic particles and living organisms on the acoustic inversion is not explicitly investigated in this study, although the extraneous scatterers could be considered as contributing to the systematic and random errors introduced into the backscattered signals used in the study.

To carry out the inversion assessment, a suspension field is simulated, sound propagated through the field and the backscattered signal calculated. It is this backscattered signal which is inverted using a number of formulations, from simple to more complex in a number of scenarios. The suspension field used is based on observations collected in coastal environments. The time-averaged vertical profile of suspended sediment concentration corresponds to a standard Rouse profile. In the absence of a general theory for vertical grain size sorting a time-averaged vertical profile based on a power law is employed. This formulation comes from observations collected in a wave dominated rippled bed environment (Thorne et al., 2011a). Although this size profile is not necessarily appropriate to highly turbulent suspension flows when size sorting may be ignored, it does offers a flow regime for testing the full performance of the acoustic profiling of both sediment concentration and grain size. Superimposed upon the mean profiles are temporal fluctuations to represent turbulence and wave motion and assessment is made as to how well these fluctuation are represented in the acoustic inversions.

The software underpinning the results presented in the paper can be found at http://noc.ac.uk/using-science/products/software/ csr-acoustic-inversions. The software consists of MATLAB programs which calculate the suspension field, the sediment 
scattering characteristics, the backscattered voltage with systematic and random errors and inversions to calculate the acoustically estimated concentration field, and the particle size and concentration fields. The software is an investigative tool for academic/ student use and is not supported by the author's institutes.

\section{Scattering formulation and inversion}

The theory of scattering from an aqueous suspension of particles has been previously presented (Sheng and Hay, 1988; Hay, 1991; Crawford and Hay, 1993; Thorne et al., 1993; Thorne and Hardcastle, 1997) and is summarised in Appendix A to provide the background to Eq. (1) below. For the normally deployed disc transceivers used for both transmission and reception, insonifying a suspension of sediments, then for the usual conditions of incoherent scattering (Morse and Ingard, 1987), the acoustically measured suspended concentration, $M$, can be related to the mean-squared backscattered voltage, $V_{m}^{2}$, as shown below (Hay and Sheng, 1992; Thorne and Hanes, 2002; Hurther et al., 2011)

$M=\left(\frac{r \psi}{K \Re}\right)^{2} V_{m}^{2} e^{4\left(r \alpha_{w}+\alpha_{s}\right)}$

where

$K=\frac{f}{\sqrt{a_{0}}}, \quad \alpha_{s}=\int_{0}^{r} \xi M d r, \quad \xi=\frac{3 \chi}{4 a_{o}}$

$\psi=\frac{1+1.35\left(r / r_{n}\right)+\left(2.5\left(r / r_{n}\right)\right)^{3.2}}{1.35\left(r / r_{n}\right)+\left(2.5\left(r / r_{n}\right)\right)^{3.2}}$

with

$f\left(x_{0}\right)=\left[\frac{\int_{0}^{\infty} \operatorname{an}(a) d a \int_{0}^{\infty} a^{2}\left(f_{i} / \sqrt{\rho}\right)^{2} n(a) d a}{\int_{0}^{\infty} a^{3} n(a) d a}\right]^{1 / 2}$

$\chi\left(x_{0}\right)=\frac{\int_{0}^{\infty} a n(a) d a \int_{0}^{\infty} a^{2}\left(\chi_{i} / \rho\right) n(a) d a}{\int_{0}^{\infty} a^{3} n(a) d a}$

$a_{o}=\int_{0}^{\infty} \operatorname{an}(a) d a$

and

$\frac{f_{i}}{\sqrt{\rho}}=\frac{\left(1-0.25 e^{-((x-1.5) / 0.35)^{2}}\right)\left(1+0.6 e^{-((x-2.9) / 1.15)^{2}}\right) x^{2}}{42+25 x^{2}}$

$\frac{\chi_{i}}{\rho}=\frac{0.09 x^{4}}{1380+560 x^{2}+150 x^{4}}$

$r$ Is the range from the transceiver and $\psi$ accounts for the departure from spherical spreading within the transducer nearfield (Downing et al., 1995), $r_{n}=\pi A_{t}^{2} / \lambda$ is the transducer nearfield, $A_{t}$ is the transducer radius, $\Re$ is the system constant incorporating the transmit and receive sensitivity, the voltage transfer function for the system, the pulse length and the directivity function of the transceiver (Betteridge et al., 2008). $K$ represents the sediment backscattering properties, $\rho$ is the sediment grain density and $a_{o}$ is the suspension mean particle radius. The term $\alpha_{\omega}$ is the sound attenuation due to water absorption and $\alpha_{s}$ is the attenuation due to suspended sediment scattering. $f_{i}$ and $\chi_{i}$ are respectively the intrinsic form function and intrinsic normalised total scattering cross-section for the particles in suspension and $x=k a$, where $k$ is the wavenumber of the sound and $a$ is the radii of the particles in suspension. Here intrinsic refers to the scattering characteristics measured using suspensions sieved into narrow $1 / 4$ phi size fractions which provide a nominally single particle size (Hay, 1991). Physically, $f_{i}$ describes the backscattering characteristics of a particle relative to its geometrical size, whilst $\chi_{i}$ quantifies the scattering from a particle over all angles, relative to its cross sectional area, and is proportional to scattering attenuation. There are a number of similar expressions for $f_{i}$ and $\chi_{i}$ (Sheng and Hay, 1988; Crawford and Hay, 1993; Thorne and Meral, 2008) and here we have chosen to present a recently developed density normalised expressions which has generic applicability to sands of varying mineralogy (Moate and Thorne, 2012). $f$ and $\chi$ represent the ensemble mean scattering values obtained by integrating the intrinsic scattering characteristics over the particle size probability density function, $n$ (a), of the particles in suspension and $x_{o}=k a_{\mathrm{o}}$. So as not to over complicate the inversions presented, particle viscous absorption (Richards et al., 2003) for $x \ll 1$, and the $\sin h(B) / B$ function (Hay, 1991; Thosteson and Hanes, 1998) required at concentrations in the tens of $\mathrm{kg} \mathrm{m}^{-3}$, are neglected. These are not particularly restrictive limitations in sandy coastal environments using low megahertz frequency ABS.

Although Eqs. (1)-(3) may appear somewhat complex, there are degrees of simplification that can be made depending on the required accuracy and detail on the suspension. These are explored for a number of cases below.

\subsection{Estimating the suspended sediment concentration}

Eq. (1) is implicit with $M$ being on either side of the equation due to the effect of attenuation by the presence of the sediments themselves. If the sediment attenuation can be assumed negligible, $r \alpha_{s} \ll 1$, the equation becomes explicit and its evaluation is simplified. Further if only the far field is considered, $\psi=1$, Eq. (1) can be written as

$M_{o}=\left(\frac{r}{K \Re}\right)^{2} V_{m}^{2} e^{4 r \alpha_{w}}$

For this case, after accounting for $\alpha_{w}$ (Clay and Medwin, 1997), the concentration at any range is simply proportional to the meansquare backscattered signal. To evaluate Eq. (4) requires a value for $K \Re$. Normally bed samples are collected when instruments are deployed and laboratory calibrations can be carried out using a suspension of the bed material. By using backscatter data collected in the far-field, at sufficiently low concentrations such that $r \alpha_{s} \ll 1$, the calibration is given by

$(K \Re)^{2}=\frac{r^{2} V_{m}^{2}}{C} e^{4 r \alpha_{w}}$

Since $C$ is the measured concentration used in the calibration and $\alpha_{w}$ can be calculated, $K \Re$ can be readily obtained. However, this calibration includes the sediment backscattering characteristics, $K$, which is site specific, based on the bed sediments and invariant with height above the bed and time. It also presupposes that the suspended sediments in the field have a size distribution equal to the bed. The approach only requires a single frequency ABS and provides a simple, though limited, calibration for estimating concentration profiles collected at a particular field site.

As mentioned above Eq. (5) provides a site specific calibration. However, if use is made of Eq. (3a) to obtain $f_{i} / \sqrt{\rho}$ and combined with a measurement of $n(a)$ of the bed, this allows $f\left(x_{o}\right)$ and $a_{o}$ to be evaluated using Eqs. (2a) and (2c) and $K$ calculated. The value for $\mathfrak{R}$ can then be estimated; this is an instrument parameter and is independent of the deployment site. With $\Re$ known the single frequency $A B S$ system can be deployed at any site and with a measurement or estimate for $n(a), f\left(x_{o}\right)$ and $a_{o}$ can be calculated, $K$ obtained for the deployment site, and inversions carried out to obtain concentration profiles, subject to the assumption of size invariance with height and time. Using a suspension of sediments to provide $\Re$ leads to a nominal value because the formulation for $f_{i} / \sqrt{\rho}$ in Eq. (3a) is a generic mean expression derived from a number of data sets (Moate and Thorne, 2012). To obtain a precise 
value for $\Re$ requires the scattering properties, and hence $K$, for the suspension to be precisely known, this can be obtained using suspensions of spheres which have an exact analytical scattering solution (Faran, 1951; Gaunaurd and Uberall, 1983; Betteridge et al., 2008).

When $\alpha_{s}$ cannot be assumed to be negligible Eq. (1) becomes implicit, with $M$ on either side of the equation and the inversion becomes slightly more complicated. If the system has been calibrated as discussed above and $K \mathfrak{R}$, or $\mathfrak{R}$ is known and $n(a)$ measured or estimated, Eq. (4) can be evaluated and $M_{o}$ profiles calculated, preferably with the nearfield correction term, $\psi$, included. An improved estimate for $M$ can be obtained using the expressions in Eq. (1) to estimate $\alpha_{s}$, by calculating $\xi$ with the formulation for $\chi_{i} / \rho$ given by Eq. (3b) with $n(a)$ to obtain $\chi$ using Eq. (2b) and evaluating

$M_{1}=M_{0} e^{4 \alpha_{s o}}$

The value for $\alpha_{\text {so }}$ is calculated using $M_{o}$. In general Eq. (6) can be written as

$M_{\kappa+1}=M_{o} e^{4 \alpha_{s \kappa}}$

Eq. (7) is iterated until a convergence criterion has been satisfied and the value for $M$ at the first range bin evaluated. The iterative process is repeated sequentially stepwise at each range bin through the $V_{m}^{2}$ profile, with the accumulating sediment attenuation accounted for and the profile of $M$ with $r$ from the transducer progressively calculated (Hay and Sheng, 1992; Thorne and Hardcastle; 1997; Thorne et al., 2011b).

\subsection{Estimating the mean particle size and suspended sediment concentration}

Apart from the site specific calibration, it has been assumed that in the evaluation of Eq. (4) the suspended particle size distribution is known or estimated and that it is invariant over the deployment period of the ABS. If the size is unknown or its temporal variability with height above the bed is required, this necessitates the ABS system to operate at more than one frequency. This is not a particular constraint as most ABS systems deployed are multi-frequency (Hay and Sheng; 1992; Thorne and Hardcastle, 1997; Villard et al., 2000; O'Hara Murray, 2012). It does, however, require $\mathfrak{R}$ to be obtained at each frequency as described above. Using the differential scattering characteristic of the suspended sediments with frequency, profiles of the acoustic measurement of particle radius, $a_{M}$, an estimate for $a_{o}$, can be made with $M$. This still requires an estimate for $n(a)$, although $a_{M}$ is calculated from the inversion. A number of approaches have been used to obtain $a_{M}$ (Hay and Sheng, 1992; Crawford and Hay, 1993; Thosteson and Hanes, 1998; Moore et al., 2013) and the approach adopted here which has been found to be relatively robust is that of Thorne et al. (2007) and Moate and Thorne (2012).

Using the approach of Thorne et al (2007), for the case when $r \alpha_{s} \ll 1, f$ is calculated using Eqs. (2a) and (3a) with $n(a)$ and $K_{j}$ evaluated at each of the $N$ frequencies in the ABS system, where subscript $j=1: N$, over a range of $a_{o}$ which covers the expected mean particle radius variation in suspension. Using these values of $K_{j}\left(a_{o}\right)$ with $V_{m j}^{2}, M_{\mathrm{oj}}\left(a_{o}\right)$ is calculated using Eq. (4) and the parameter $\Phi$ evaluated for each value of $a_{o}$ over the range of $a_{o}$ as below

$\Phi=\frac{\sigma_{M}\left(a_{o}\right)}{M_{o}\left(a_{o}\right)}$

$M_{o}\left(a_{o}\right)=\frac{1}{N} \sum_{j=1}^{N} M_{o j}\left(a_{o}\right) \quad \sigma_{M}^{2}\left(a_{o}\right)=\frac{1}{N} \sum_{j=1}^{N} M_{o j}^{2}\left(a_{o}\right)-\left[M_{o}\left(a_{o}\right)\right]^{2}$

The value of $a_{o}$ for which $\Phi$ is a minimum at each range gate provides profiles of $a_{M}$ and $\mathrm{M}_{\mathrm{o}}$ over the deployment period.
Illustrations of this process and the form of $\Phi$ are presented in Appendix B. When $\alpha_{s}$ is not negligible the additional complication of an implicit iterative inversion is required. The procedure uses Eqs. (4), (6) and (7), with $\chi$ calculated using Eqs. (2b) and (3b) with $n(a)$, for the range of $a_{o}$, at each frequency, and Eq. (8) evaluated with $M$ replacing $M_{o}$. The implicit iterative process is repeated sequentially stepwise at each range bin through the $V_{m j}^{2}$ profiles, with the accumulating sediment attenuation accounted for and the profiles of $a_{M}$ and $M$ with $\mathrm{r}$ from the transducer progressively calculated (Thorne et al., 2011b). This is the full inversion of Eq. (1) when the temporal-spatial structure of $a_{M}$ and $M$ for the suspension field is required.

\subsection{Scattering properties and sediment size distribution}

In Eq. (3) the density normalized intrinsic scattering properties, $f_{i} / \sqrt{\rho}$ and $\chi_{i} \mid \rho$ are given, however, invariably, in the marine environment, there will be a particle size distribution and the ensemble scattering characteristics described in Eq. (2), $f$ and $\chi$, are required. It is therefore important to assess how $n(a)$ impacts on the suspension scattering characteristics. To assess this three common probability density functions for the size were examined; the normal, $n_{n}(a)$, lognormal, $n_{l}(a)$, and bi-normal, $n_{b i}(a)$. These are respectively given by;

$n_{n}(a)=\frac{1}{\sigma \sqrt{2 \pi}} e^{-\left(a-a_{0}\right)^{2} / 2 \sigma^{2}}$

$n_{l}(a)=\frac{1}{a \zeta \sqrt{2 \pi}} e^{-\left(\log _{e}(a)-\gamma\right)^{2} / 2 \zeta^{2}}, \zeta=\sqrt{\log _{e}\left[\left(\sigma / a_{o}\right)^{2}+1\right]}$,

$\gamma=\log _{e}\left(a_{o}^{2} / \sqrt{a_{o}^{2}+\sigma^{2}}\right)$

$n_{b i}(a)=\frac{1}{\sigma_{1} \sqrt{2 \pi}} e^{-\left(a-a_{1}\right)^{2} / 2 \sigma_{1}^{2}} \frac{1}{\sigma_{2} \sqrt{2 \pi}} e^{-\left(a-a_{2}\right)^{2} / 2 \sigma_{2}^{2}}$

For all the distributions the parameters were chosen so that the normalised standard deviation, $\delta=\sigma / a_{o}$, of $n(a)$ was equal to $\delta=0.4$. A comparison of the distributions are shown in Fig. 1a for a value of $a_{o}=100 \mu \mathrm{m}$. The ensemble scattering characteristics $f$ and $\chi$ were calculated using Eq. (2), by integrating the density normalized intrinsic values, $f_{i} / \sqrt{\rho}$ and $\chi_{i} / \rho$ given in Eq. (3), over $n(a)$ described in Eqs. (9a)-(9c), and the scattering and attenuation parameters $K$ and $\xi$ evaluated. The results are shown in Fig. $1 \mathrm{~b}$ and $\mathrm{c}$ for a frequency of $2.0 \mathrm{MHz}$; increasing or decreasing the frequency respectively raises or lowers the values of $K$ and $\xi$, however, the form with $x_{0}$ remains constant. The solid line represents the intrinsic scattering characteristics and the symbols the ensemble scattering characteristics. As can be seen the impact of the size distribution on the scattering characteristics is to substantially increase values in the Rayleigh regime, $x_{0} \ll 1$, while marginally reducing values in the geometric regime, $x_{o} \gg 1$ (Thorne and Meral, 2008). The departure from the intrinsic scattering properties is similar for the three distributions and scales with the standard deviation. Therefore to first order it is the value for $\sigma$ which controls the departure from the intrinsic scattering properties, rather than the precise form of $n(a)$. This divergence of the ensemble scattering characteristic from the intrinsic will impact on acoustic inversions and therefore needs to be accounted for when calculating particle size and concentration from acoustic backscattered data.

\subsection{Statistics of the backscattered signal}

Eq. (1) provides an expression for $M$ based on incoherent scattering due to the backscattered signal having random phase statistics uniformly distributed over $2 \pi$. To obtain $V_{m}^{2}, V^{2}$ is average over a number of independent backscattered signals and the 
a

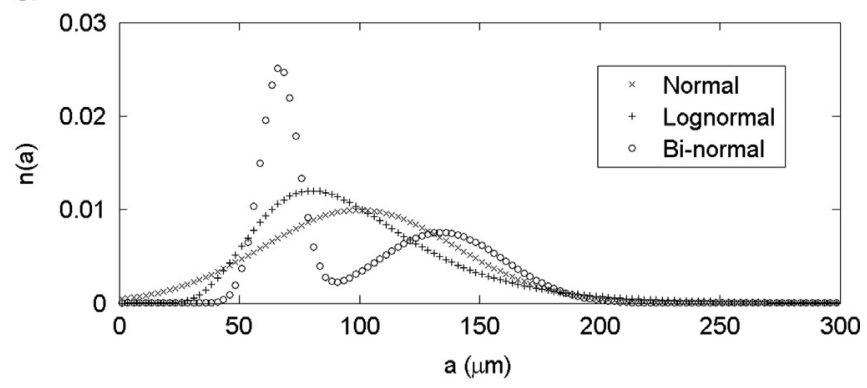

b

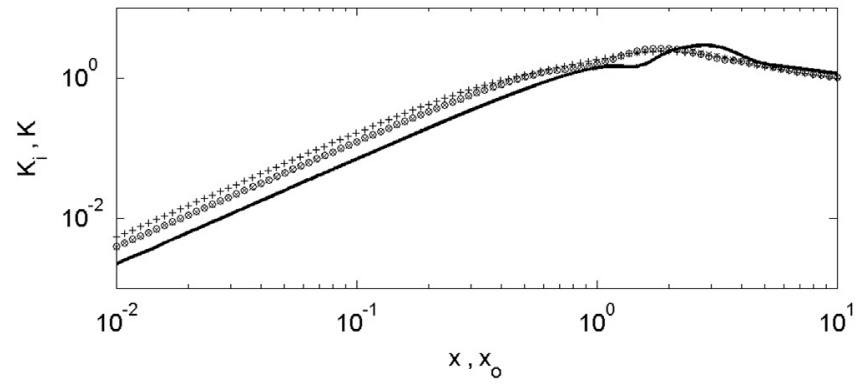

C

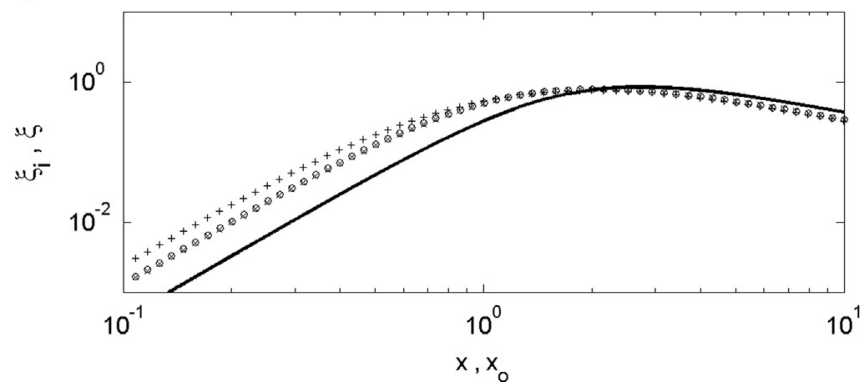

Fig. 1. (a) Probability density function of the particle size number, $n(a)$, for normal, lognormal and bi-normal distributions. ((b) and (c)) Impact on the backscattering, $K$, and attenuation, $\xi$, scattering characteristics due to a size distribution. The solid line represents intrinsic values and the symbols ensemble values integrated over the three distributions for $n(a)$ shown in (a).

number averaged determines the standard error for $V_{m}^{2}$. For incoherent scattering the probability distribution for the backscatter signal, $V$, is Rayleigh (Hay, 1983; Thorne et al., 1993) and for $V^{2}$ exponential (Wilhelmij and Denbigh, 1984; Libicki et al., 1989)

$p\left(V^{2}\right)=\beta e^{-\left(\beta V^{2}\right)}$

where $\beta=1 / V_{m}^{2}$. For an exponential distribution the standard deviation, $\sigma=1 / \beta$, therefore the standard error in $V_{m}^{2}$ is given by

$\sigma_{e}\left(V_{m}^{2}\right)=\frac{V_{m}^{2}}{\sqrt{\eta}}$

where $\eta$ is the number of independent profiles averaged to form $V_{m}^{2}$. Since $M$ is proportional to $V_{m}^{2}$ the normalized standard error in the estimated concentration due to the statistical nature of the backscattered signal is $\sigma_{e}(M) / M \approx 1 / \sqrt{\eta}$. To assess these statistical characteristics, Fig. 2a and b shows results from backscatter data collected on a homogeneous suspension of $1 / 4 \varphi$ sieved magnetite sand of diameter $275 \mu \mathrm{m}$, at frequencies of $1.0(\bullet), 2.93(0), 4.02(\Delta)$ and $4.9(+) \mathrm{MHz}$, with a pulse length of $10 \mu$ s and pulse repetition frequency, prf, of $4 \mathrm{~Hz}$; moderately sorted suspended quartz sand, $d_{50}=240 \mu \mathrm{m} \sigma(d)=83 \mu \mathrm{m}$, under waves with a prf of $160 \mathrm{~Hz}$, pulse length of $4 \mu \mathrm{s}$ and frequency of $1.25 \mathrm{MHz}$, and from flowing water a

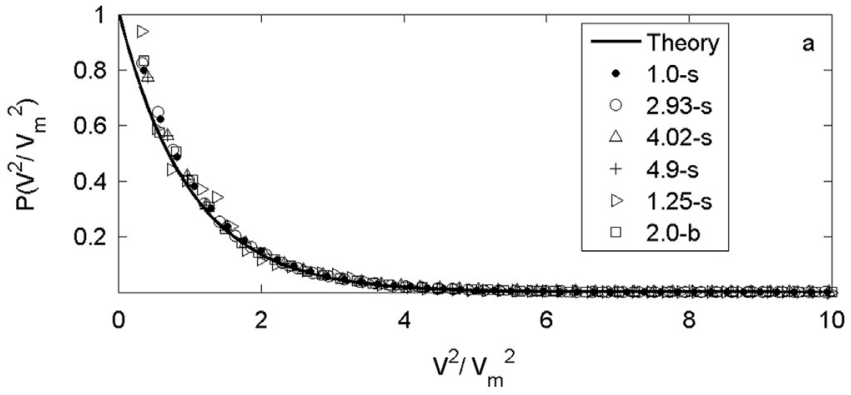

b

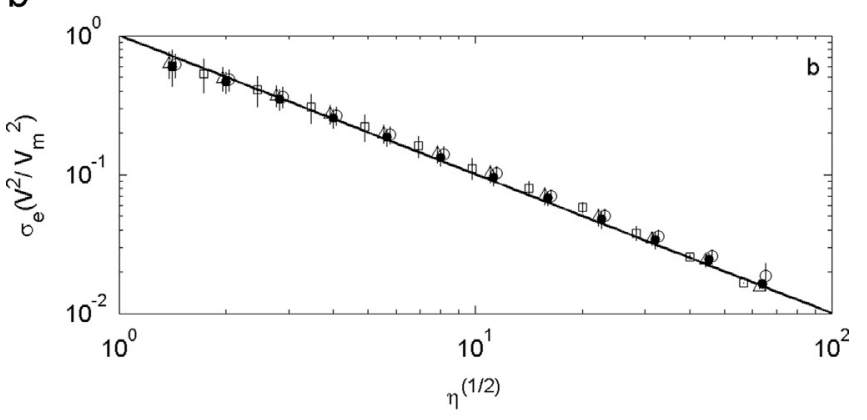

C

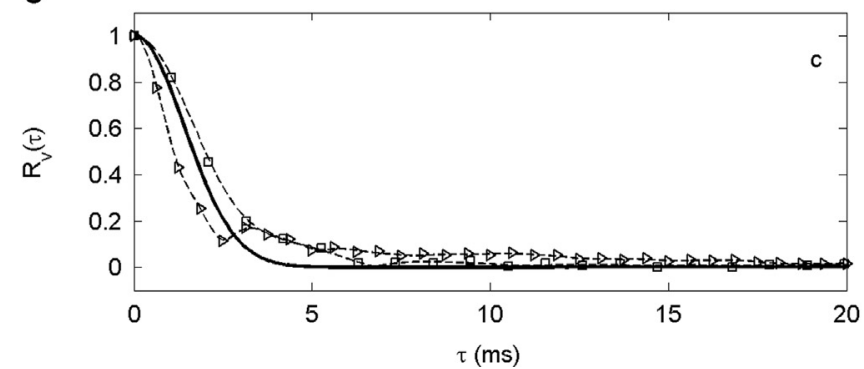

Fig. 2. Measurement of the backscattered statistics. (a) Normalised backscattered squared voltage probability density function, (b) normalised standard error and (c) autocorrelation function of the backscattered signal amplitude. In the legend in (a) the numbers refer to frequency in megahertz and the letters to scatterer type, $s$ sand, $b$-bubbles, and the nomenclature applies to the three plots.

containing nominally homogeneous micro-bubbles in a open channel with a prf of $95 \mathrm{~Hz}$, a pulse length of $2 \mu \mathrm{s}$ and a frequency of $2.0 \mathrm{MHz}$. Each data set was normalised by its mean square value so that the results could be compared with a single theoretical prediction, given by the solid line in the figures. As can be seen, for the varied frequencies, pulse repetition frequencies, pulse lengths and scatterer type, the data follow the theoretical predictions, thereby confirming the assumption of incoherent scattering upon which the ABS theoretical analysis is formulated.

The value for $\eta$ used to assess the accuracy of $M$ is based on independent samples and therefore it is important to ascertain the decorrelation time to obtain independent samples. To examine the decorrelation, the signal amplitude from an acoustic Doppler velocity profiler (Hurther et al., 2011) backscattered from suspended sediments under waves and flowing water containing micro-bubbles, were collected using at a pulse repetition frequency respectively of $1600 \mathrm{~Hz}$ and $952.4 \mathrm{~Hz}$. The measured autocorrelation functions of the backscatter signals with time lag are shown in Fig. 2c. The figure clearly shows an initial reduction in the level of the autocorrelation functions followed by a nominally constant value close to zero. The solid line in the plot is a simple empirical fit to the data obtained using $e^{\left(-\tau / \tau_{0}\right)^{2}}$ where $\tau$ is the lag and $\tau_{\mathrm{o}}=2.0 \mathrm{~ms}$. The present results indicate a 
decorrelation time of around 3-5 ms. Hay and Bowen (1994) using a $2.0 \mathrm{MHz}$ ABS under waves in the field estimated a decorrelation time of 2-4 ms. Therefore if an upper limit of $5 \mathrm{~ms}$ is assumed for the decorrelation time, the corresponding maximum pulse repetition frequency for independent samples would be $200 \mathrm{~Hz}$. If $5.0 \mathrm{~Hz}$ concentration profiles were required an accuracy of $\sigma(M) / M \approx 0.16$ would result from backscattered profiles collected at $200 \mathrm{~Hz}$. If higher accuracy was needed then vertical averaging over independent range bins could be carried out. The importance of the statistical nature of the backscatter signal, and the requirement for averaging over independent samples, is that it may lead to a degree of compromise between the desired spatial-temporal resolution and the degree of averaging necessary to obtain an acceptable accuracy for $M$.

\section{Backscattering from a suspension field}

\subsection{Formulation of the suspended field}

To assess the different inversions described in Section 2 requires simulations to be carried out. Therefore to examine the inversion of the backscattered signal and the resulting acoustic estimates of suspended sediment parameters a simulated suspension field through which the sound could be propagated was required. To establish the suspension field the underlying profiles for the time averaged concentration, $\bar{C}$, and the time averaged mean particle radius, $\overline{a_{c}}$ were of the form

$\bar{C}=\overline{C_{r}}\left(\frac{z}{z_{r}}\right)^{-p}$

$\overline{a_{c}}=\overline{a_{r}}\left(\frac{z}{z_{r}}\right)^{-l}$

Eq. (12a) is the commonly used Rouse profile (Soulsby, 1997) which approximates to a power law near the bed, $p$ is the Rouse parameter $p=w_{s} / \kappa u^{*}$, where $u^{*}$ is the bed friction velocity, $\kappa$ is the Von Karman constant and $w_{s}$ is the particle settling velocity. $\overline{C_{r}}$ is the reference concentration and for the present study it was specified at the lowest ABS range bin above the bed which was at a height of $z_{r}=0.005 \mathrm{~m}$. In the absence of a general theory for the vertical grain size sorting effects and the lack of size sorting measurements in the literature, it is adopted here that $\overline{a_{c}}$ follows the power law given by Eq. (12b). This is based on the observations (Thorne et al., 2011a) collected over a rippled sand bed for a range of wave forcing and bed sediment size conditions. For this type of flow, grain size sorting effects are found to be significant resulting in decreasing $\overline{a_{c}}$ corresponding with the region of the Rouse profile. It is acknowledged that such a form is not generic, for example under highly turbulent conditions for fine sediments $\overline{a_{c}}$ profiles may be more uniform. Further, vertical temporal differential settling velocities within the suspended size distribution are not specifically addressed in the simulated suspension field. However, the selected ripple suspension conditions are appropriate for testing the full performance of the acoustic profiling of both sediment concentration and grain size when size sorting effects are important. For the present study the variables in Eq. (12) were given the following values; $p=1, \quad l=0.2, \overline{C_{r}}=5.0 \mathrm{~kg} \mathrm{~m}^{-3}$ and $\overline{a_{c}}=78 \mu \mathrm{m}$.

As well as requiring a time averaged mean size profile, a size distribution was also required to represent the suspension. Most sedimentologists use a mass probability density function, $m(a)$, to represent particle size and one of the more common distributions fitted to natural sediments is the lognormal given in Eq. (9b) (Soulsby, 1997). For the acoustic analysis the probability density

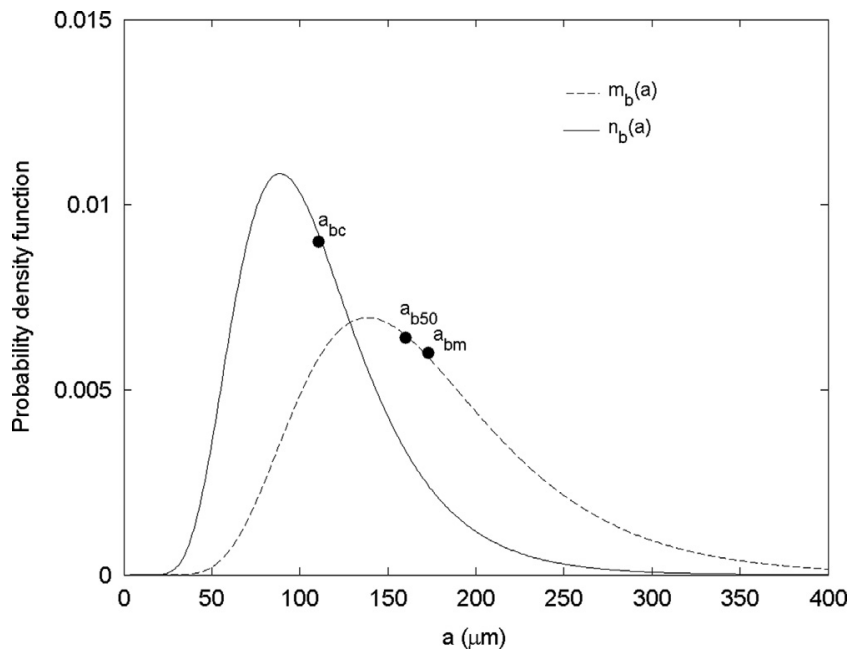

Fig. 3. The probability density function for the bed mass size, $m_{b}(a)(---)$, and the number size, $n_{b}(\mathrm{a})(-)$, used for the acoustic inversions. $a_{\mathrm{bm}}(173 \mu \mathrm{m})$ is the mean mass radius, $a_{\mathrm{b} 50}(160 \mu \mathrm{m})$ is the median mass radius and $a_{\mathrm{bc}}(110 \mu \mathrm{m})$ is the mean number radius.

function required is not $m(a)$, but $n(a)$, which is obtained from $m(a) / a^{3}$ assuming nominally spherically shaped particles. In Fig. 3 is shown the lognormal bed sediment mass probability density function, $m_{b}(a)$, used in the present study, from which the suspended sediment profiles were derived. The distribution had $\delta_{b}=0.4$, where $\delta_{b}=\sigma_{\mathrm{bm}} / a_{\mathrm{bm}}$, and $\sigma_{\mathrm{bm}}$ and $a_{\mathrm{bm}}$ are respectively the standard deviation and mean of $m_{b}(a)$. For the suspended sediments $\delta$ was also set to a value 0.4 and therefore as $a_{c}$ reduced with height above the bed $\sigma_{\mathrm{c}}$ proportionally decreased. For the bed $a_{\mathrm{bm}}=173 \mu \mathrm{m}$ and the median grain diameter $2 a_{\mathrm{b} 50}=d_{\mathrm{b} 50}=320 \mu \mathrm{m}$. Based on the Wentworth scale (Soulsby, 1997) this is medium sand. The form for $n_{b}(a)$ is also shown in Fig. 3, it is down shifted in radius with its mean value, $a_{\mathrm{bc}}$, being $a_{\mathrm{bc}}=0.64 a_{\mathrm{bm}}(110 \mu \mathrm{m})$, although $n_{b}(a)$ remains lognormal distributed and retains the same ratio of the standard deviation, $\sigma_{\mathrm{bc}}$, to mean, $\sigma_{\mathrm{bc}}=0.4 a_{\mathrm{bc}}$. The radius which is measured acoustically, $a_{M}$, is an estimate for $a_{c}$ and this has implications for sediment transport studies. Marine scientists generally use $d_{50}$, based on $m(a)$, to designate the size of the particles in suspension and it is $d_{50}$ upon which most sediment transport formulations are based (Soulsby, 1997). In the present case $\sigma_{\mathrm{bc}}=0.4 a_{\mathrm{bc}}, a_{\mathrm{bc}}=0.64 a_{\mathrm{bm}}$, and $d_{\mathrm{b} 50}=2.9 a_{\mathrm{bc}}$. Therefore the mean particle diameter, $2 a_{M}$, obtained from an acoustic inversion, which is an estimate for $2 a_{c}$, is not the size used in most sediment formulae; this point is returned to in Section 5.4.

Eq. (12) provides the basic formulation for the time averaged suspension field, however to represent the temporal and spatial variability, arising from turbulent and intra-wave fluctuations in the hydrodynamics, periodic and random components were superimposed upon the basic structure. The results for the $a_{c}$ and $C$ fields are shown in Fig. 4a and d. The temporal and vertical variability portrayed in these figures is considered to be consistent with observations made in the coastal zone (Hay and Bowen, 1994; O'Hara Murray, 2012). The degree of coherence between $a_{c}$ and $C$ is associated with more dynamic events generating higher concentrations with larger particles sizes, while weaker events have lower concentrations and smaller suspended particles Fig. 4b and e show the mean profile averaged over the record length, $\overline{a_{c}}$ and $\bar{C}$ and Fig. 4c and f shows the time series reference values at $z=0.005 \mathrm{~m}$. The reference values varied between $C_{r}=0.7-$ $16 \mathrm{~kg} \mathrm{~m}^{-3}$ and $a_{r}=57-110 \mu \mathrm{m}$. It is the suspension fields shown in Fig. 4a and b, combined with the lognormal size distribution shown in Fig. 3, which are used in the present analysis. 

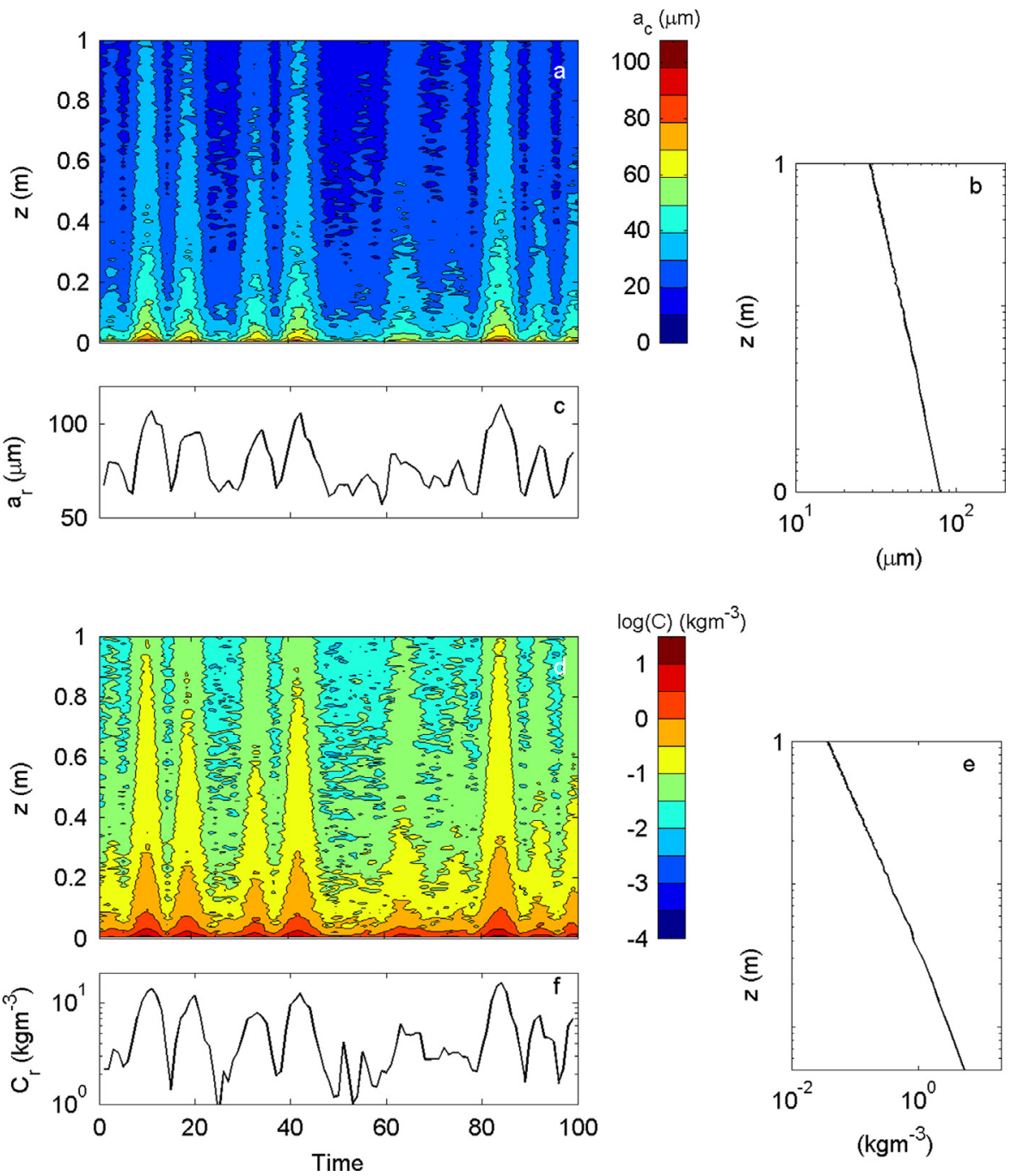

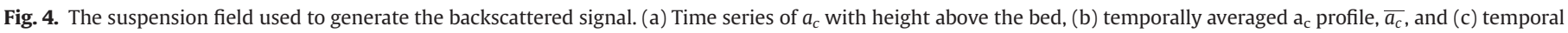
variation of $a_{r}$ at $z_{r}$. (d) Time series of $C$ with height above the bed, (e) temporally averaged $C$ profile, $\bar{C}$, and (f) temporal variation of $C_{r}$ at $z_{r}$.

In Fig. 4c the peak value for $a_{r}=110 \mu \mathrm{m}$ and this is based on the value of $d_{\mathrm{b} 50}$ for the bed sediments shown in Fig. 3. Since the bed sediments have a $d_{\mathrm{b} 50}=320 \mu \mathrm{m}$, then if all the bed sediments were entrained the suspended $d_{50}$ would be $320 \mu \mathrm{m}$ and as noted above for $\delta=0.4, a_{\mathrm{bc}}=d_{\mathrm{b} 50} / 2.9$, therefore it follows the maximum value for $a_{r}$ will be $110 \mu \mathrm{m}$. However, the condition for all size fractions being entrained occurs only during the most energetic events and in general, as shown in Fig. 4c, the suspended sediments have a size fraction which is smaller than the bed and this reduces with height above the bed following the form shown in Fig. $4 \mathrm{~b}$ and given by Eq. (12b). This size sorting has implications for the acoustic inversions to be discussed.

\subsection{The forward problem; calculating the backscattered signal for the inversions}

Using the suspended sediment time series presented in Fig. 4a and $\mathrm{d}$, with the specified lognormal distribution for $n(a)$ presented in Fig. 3, the forward problem of propagating sound through the suspension field was solved using Eq. (A1.4) in Appendix A and the backscattered signal calculated. First backscatter signals were calculated with no uncertainties. Second, systematic errors and random fluctuations were introduced into the backscattered signal via Eq. (A1.4), due to variability in the scattering characteristics
$K=\varepsilon K\left(1 \pm \gamma_{\mathrm{s}}\right)$ and $\xi=\varepsilon \xi\left(1 \pm \gamma_{\mathrm{s}}\right)$, the statistical nature of the backscattered signal $V_{\text {rms }}=V_{\text {rms }}\left(1 \pm \gamma_{\mathrm{v}}\right)$ and calibration uncertainties $\Re=\varepsilon \Re\left(1 \pm \gamma_{\mathfrak{R}}\right)$. $\varepsilon$ was a scaling factor either greater or less than unity and independent of range from the transducer and time. $\gamma_{s}$, $\gamma_{\mathrm{v}}$ and $\gamma_{\mathfrak{R}}$ had random values with range and time, that lay between 0 and $\gamma_{0}$ and were uncorrelated between the parameters. When a multi-frequency system was simulated the random components were also uncorrelated between frequencies. The time averaged value for the random components was zero. The lack of adding a systematic error to $V_{\text {rms }}$ was because this was introduced through $\mathfrak{R}$. These computed backscattered signals were used in different inversion scenarios based on Eq. (1) and its simplification and the calculated acoustic estimates for the concentration, $M$, and particle radius, $a_{M}$, compared with $C$ and $a_{c}$ shown in Fig. 4.

\section{Inversion scenarios}

In this section a number of inversions scenarios of increasing complexity are described. These are broadly in terms of the type of ABS used, whether it be single or multi-frequency, the underlying assumptions made regarding the suspension structure, how supporting independent data can be used to aid the acoustic inversions and an assessment of the impact systematic and random 
Table 1

Scenarios used in the single frequency inversion scenarios. Explanations for the parameters are given in the text.

\begin{tabular}{|c|c|c|c|c|c|c|c|c|c|c|c|c|c|}
\hline \multirow[t]{3}{*}{ Scenario } & \multirow{3}{*}{$\begin{array}{l}\text { ABS } \\
\text { type }\end{array}$} & \multirow{3}{*}{$\begin{array}{l}\text { Inv. } \\
\text { method }\end{array}$} & \multirow{3}{*}{$\begin{array}{l}\text { Avail. sed. } \\
\text { samples }\end{array}$} & \multirow{3}{*}{$\begin{array}{l}\text { Calc. } \\
\text { prof. }\end{array}$} & \multicolumn{6}{|c|}{ Inversion assumptions } & \multicolumn{2}{|c|}{ Uncertainties } & \multirow[t]{3}{*}{ Shown results } \\
\hline & & & & & \multicolumn{4}{|c|}{ Sediment parameter } & \multicolumn{2}{|c|}{$\begin{array}{l}\text { Syst. } \\
\text { param. }\end{array}$} & \multirow{2}{*}{$\begin{array}{l}\text { Systematic } \\
\text { error } \\
\varepsilon\end{array}$} & \multirow{2}{*}{$\begin{array}{l}\begin{array}{l}\text { Random } \\
\text { error }\end{array} \\
\gamma\end{array}$} & \\
\hline & & & & & $n(a) a_{o}$ & $K$ & $\xi$ & $\alpha_{S}$ & $\Re$ & $\psi$ & & & \\
\hline SF1a & $\begin{array}{l}\text { Single } \\
\text { freq. } \\
2 \mathrm{MHz}\end{array}$ & $\begin{array}{l}\text { Eq. (4). } \\
\text { Explicit }\end{array}$ & No & $M$ & $-a_{b 50}$ & $K_{i}\left(a_{b 50}\right)$ & - & 0 & $\Re$ & 1 & 1 & 0 & $\begin{array}{l}\text { Fig. } 5 \text { a (field) Fig. 5b-d, } \\
\text { solid circles }\end{array}$ \\
\hline SF1b & $\begin{array}{l}\text { Single } \\
\text { freq. } \\
2 \mathrm{MHz}\end{array}$ & $\begin{array}{l}\text { Eq. (4) } \\
\text { Explicit }\end{array}$ & No & $M$ & $-a_{b 50}$ & $K_{i}\left(a_{b 50}\right)$ & - & 0 & $\Re$ & 1 & $0.8 \& 1.2$ & 0.1 & $\begin{array}{l}\text { Fig. 5b, c (timeseries) } \\
\text { Fig. } 5 d \text { (mean profile) } \\
\text { Grey shaded }\end{array}$ \\
\hline SF1c & $\begin{array}{l}\text { Single } \\
\text { freq. } \\
2 \mathrm{MHz}\end{array}$ & $\begin{array}{l}\text { Implicit } \\
\text { iter. Eq. } \\
(7)\end{array}$ & No & $M$ & $-a_{b 50}$ & $K_{i}\left(a_{b 50}\right)$ & $\xi_{i}=\xi_{i}\left(a_{b 50}\right)$ & $\xi_{i} \int_{0}^{z} M(z, t) d z$ & $\mathfrak{R}$ & $\psi$ & 1 & 0 & $\begin{array}{l}\text { Fig. 5d (mean profile) } \\
\text { Dashed line }\end{array}$ \\
\hline SF2a & $\begin{array}{l}\text { Single } \\
\text { freq. } \\
2 \mathrm{MHz}\end{array}$ & $\begin{array}{l}\text { Eq. (4) } \\
\text { Explicit }\end{array}$ & Bed sample & $M$ & $n_{b}, a_{b c}$ & $K\left(a_{b c}\right)$ & - & 0 & $\Re$ & 1 & 1 & 0 & $\begin{array}{l}\text { Fig. 6a (field) Fig. 6b-d } \\
\text { solid circles }\end{array}$ \\
\hline SF2b & $\begin{array}{l}\text { Single } \\
\text { freq. } \\
2 \mathrm{MHz}\end{array}$ & Eq. (4) & Bed sample & M & $n_{b}, a_{b c}$ & $K\left(a_{b c}\right)$ & - & 0 & $\mathfrak{R}$ & 1 & $0.8 \& 1.2$ & 0.1 & $\begin{array}{l}\text { Fig. 6b, c (timeseries) } \\
\text { Fig. 6d (mean profile) } \\
\text { Grey shaded }\end{array}$ \\
\hline SF2c & $\begin{array}{l}\text { Single } \\
\text { freq. } \\
2 \mathrm{MHz}\end{array}$ & $\begin{array}{l}\text { Implicit } \\
\text { iter. Eq. } \\
(7)\end{array}$ & Bed sample & $M$ & $n_{b}, a_{b c}$ & $K\left(a_{b c}\right)$ & $\xi_{b}=\xi\left(a_{b c}\right)$ & $\xi_{b} \int_{0}^{z} M(z, t) d z$ & $\Re$ & $\psi$ & 1 & 0 & $\begin{array}{l}\text { Fig. 6d (mean profile) } \\
\text { Dashed line }\end{array}$ \\
\hline SF3a & $\begin{array}{l}\text { Single } \\
\text { freq. } \\
2 \mathrm{MHz}\end{array}$ & $\begin{array}{l}\text { Eq. (4) } \\
\text { Explicit }\end{array}$ & $\begin{array}{l}a_{o}(z) \text { Assumed } \\
+ \text { bed sample }\end{array}$ & $M$ & $\begin{array}{l}n_{b} \bar{a}_{c}(z) \\
\text { with } \\
\bar{a}_{c}\left(z_{r}\right)=a_{b c}\end{array}$ & $K\left(\bar{a}_{c}(z)\right)$ & - & 0 & $\Re$ & 1 & 1 & 0 & $\begin{array}{l}\text { Fig. 7a (field) Fig. 7b-d } \\
\text { Solid circles }\end{array}$ \\
\hline SF3b & $\begin{array}{l}\text { Single } \\
\text { freq. } \\
2 \mathrm{MHz}\end{array}$ & $\begin{array}{l}\text { Eq. (4) } \\
\text { Explicit }\end{array}$ & $\begin{array}{l}a_{o}(z) \\
\text { Assumed+bed } \\
\text { sample }\end{array}$ & $M$ & $\begin{array}{l}n_{b} \bar{a}_{c}(z) \\
\text { with } \\
\bar{a}_{c}\left(z_{r}\right)=a_{b c}\end{array}$ & $K\left(\bar{a}_{c}(z)\right)$ & - & 0 & $\Re$ & 1 & $0.8 \& 1.2$ & 0.1 & $\begin{array}{l}\text { Fig. 7b, c (timeseries) } \\
\text { Fig. 7d (mean profile) } \\
\text { Grey shaded }\end{array}$ \\
\hline SF3c & $\begin{array}{l}\text { Single } \\
\text { freq. } \\
2 \mathrm{MHz}\end{array}$ & $\begin{array}{l}\text { Implicit } \\
\text { iter. Eq. } \\
(7)\end{array}$ & $\begin{array}{l}a_{o}(z) \\
\text { Assumed + bed } \\
\text { sample }\end{array}$ & $M$ & $\begin{array}{l}n_{b} \bar{a}_{c}(z) \\
\text { with } \\
\bar{a}_{c}\left(z_{r}\right)=a_{b c}\end{array}$ & $K\left(\bar{a}_{c}(z)\right)$ & $\xi\left(\bar{a}_{c}(z)\right)$ & $\int_{0}^{z} \xi\left(\bar{a}_{c}(z)\right) M(z, t) d z$ & $\Re$ & $\psi$ & 1 & 0 & $\begin{array}{l}\text { Fig. } 7 d \text { (mean profile) } \\
\text { Dashed line }\end{array}$ \\
\hline
\end{tabular}

errors have on the acoustic estimates of $a_{c}$ and $C$. Tables 1 and 2 provide an overview of the conditions for all the cases simulated which are outlined below. In Section 5, the calculated acoustic values for $M$ and $a_{M}$ for the varying scenarios are presented.

\subsection{Single frequency, SF, cases; SF1, SF2, SF3 in Table 1}

For the first three cases named SF1, SF2 and SF3 in Table 1, a single frequency $\mathrm{ABS}$ operating at $2 \mathrm{MHz}$ mounted at $1.0 \mathrm{~m}$ above the bed with a vertical spatial resolution of $0.005 \mathrm{~m}$ was examined. Systematic, $\varepsilon$, and random, $\gamma_{\mathrm{o}}$, uncertainties were introduced into the forward calculation through Eq. (A1.4) and in the inversions assumptions were made about the suspended particle size, the effect of acoustic attenuation and the transducer nearfield directivity. Since a single frequency was employed these inversions only calculated profiles of suspended mass concentration, $M$, for the conditions described in Section 3.1.

Case SF1 corresponds to a practical situation where only the $d_{\mathrm{b} 50}$ value of the bed sediments was known. The inversion was therefore evaluated using $a_{o}=d_{\mathrm{b} 50} / 2(160 \mu \mathrm{m})$, the intrinsic functions for $K_{i}$ and $\xi_{\mathrm{i}}$ and with no grain size sorting with height above the bed and in time. The concentration profiles for $M$ were calculated for three inversions scenarios. SF1a where no uncertainties were introduced into the forward calculation of the backscattered signal, $\varepsilon=1, \gamma_{\mathrm{o}}=0$ and in the inversion sediment attenuation was assumed negligible, $r \alpha_{s} \ll 1$, and the nearfield correction was ignored, $\psi=1$. SF1b where uncertainties, $\varepsilon=0.8$ and $\varepsilon=1.2$ and $\gamma_{\mathrm{o}}=0.1$ were introduced into the calculation of the backscattered signal and again in the inversion it was assumed $r \alpha_{s} \ll 1$ and $\psi=1$. SF $1 \mathrm{c}$ with no uncertainties, $\varepsilon=1$ and $\gamma_{\mathrm{o}}=0$, being used in the calculation for the backscattered signal, and with $\alpha_{s}$ and $\psi$ being accounted for in the inversion. In this latter case, the iterative implicit inversion had to be applied for the calculation of $M$ profiles.

In the case SF2, further sediment size information was available in the form of a bed sample. The inversion was therefore evaluated using the lognormal number density distribution function of the bed, $n_{b}(a)$, to calculate the ensemble mean values of the sediment parameters, $a_{o}=a_{\mathrm{bc}}(110 \mu \mathrm{m}), K$ and $\xi$ with no grain size sorting with height above the bed and in time. The concentration profile $M$ was calculated for three inversion scenarios named SF2a, SF2b, SF2c which were the same in terms of uncertainty, attenuation and nearfield conditions to scenarios SF1a, SF1b and SF1c. For the third case, SF3, some information on the suspended sediments was considered available. This case simulates the situation when samples of the suspended sediments were collected or an assumption was made for the time averaged suspended size profile based on the bed sediments. In the latter case $a_{o}=a_{\mathrm{bc}}\left(z \mid z_{r}\right){ }^{-l} l=0.2$, and $K$ and $\xi$ were a function of height above the bed, $z$, however, they were still time invariant. The same three scenarios of uncertainty, attenuation and nearfield conditions for SF1, SF2 were applied to case SF3.

\subsection{Multi-frequency, MF, cases; MF1, MF2, MF3 in Table 2}

The utilisation of a multi-frequency ABS system provides the opportunity to estimate vertical profiles of both the $M$ and $a_{M}$ as a function of time. For the cases studied here the ABS had frequencies of $0.7,1.25$ and $3.0 \mathrm{MHz}$, was mounted $1 \mathrm{~m}$ above the bed and had a vertical resolution of $0.005 \mathrm{~m}$. To carry out the multifrequency inversion requires an estimate for the form of $n(a)$ so that $K$ and $\xi$ can be calculated in the inversion. $n(a)$ was chosen to be the same as in the forward calculations based on the bed lognormal sediments size distribution shown in Fig. 3 with 
Table 2

Scenarios used in the multi-frequency inversion scenarios. Explanations for the parameters are given in the text.

\begin{tabular}{|c|c|c|c|c|c|c|c|c|c|c|c|c|c|}
\hline \multirow[t]{3}{*}{ Scenario } & \multirow{3}{*}{$\begin{array}{l}\text { ABS } \\
\text { type }\end{array}$} & \multirow{3}{*}{$\begin{array}{l}\text { Inv. } \\
\text { method }\end{array}$} & \multirow{3}{*}{$\begin{array}{l}\text { Avail. sed. } \\
\text { samples }\end{array}$} & \multirow{3}{*}{$\begin{array}{l}\text { Calc. } \\
\text { prof. }\end{array}$} & \multicolumn{6}{|c|}{ Inversion assumptions } & \multicolumn{2}{|c|}{ Uncertainties } & \multirow[t]{3}{*}{ Shown results } \\
\hline & & & & & \multicolumn{4}{|c|}{ Sediment parameter } & \multicolumn{2}{|c|}{$\begin{array}{l}\text { Syst. } \\
\text { param. }\end{array}$} & \multirow{2}{*}{$\begin{array}{l}\begin{array}{l}\text { Systematic } \\
\text { error }\end{array} \\
\varepsilon\end{array}$} & \multirow{2}{*}{$\begin{array}{l}\begin{array}{l}\text { Random } \\
\text { error }\end{array} \\
\gamma_{o}\end{array}$} & \\
\hline & & & & & $n(a)$ & $K$ & $\xi$ & $\alpha_{s}$ & $\Re$ & $\psi$ & & & \\
\hline MF1 & $\begin{array}{l}\text { Multi } \\
\text { freq. } \\
0.7 / \\
1.25 / \\
3 \mathrm{MHz}\end{array}$ & $\begin{array}{l}\text { Explicit. } \\
\text { Eq. }(8)\end{array}$ & $\begin{array}{l}n(a) \\
\text { Assumed } \\
\text { or bed } \\
\text { sample }\end{array}$ & $\begin{array}{l}M \\
\text { and } \\
a_{M}\end{array}$ & $\begin{array}{l}n\left(a_{o}, z, t\right) \\
a_{o}=2-400 \mu m\end{array}$ & $K_{j}\left(a_{o}, z, t\right)$ & - & 0 & $\Re_{\mathrm{j}}$ & 1 & 1 & 0 & $\begin{array}{l}\text { Fig. 8a \& b (mean profile) } \\
\text { Fig. 8c \& d (fields) Fig. 8e \& f } \\
\text { (timeseries) Solid circles }\end{array}$ \\
\hline MF2a & $\begin{array}{l}\text { Multi } \\
\text { freq. } \\
0.7 / \\
1.25 / \\
3 \mathrm{MHz}\end{array}$ & $\begin{array}{l}\text { Implicit } \\
\text { iter. Eq. } \\
(8)\end{array}$ & $\begin{array}{l}n(a) \\
\text { Assumed } \\
\text { or bed } \\
\text { sample }\end{array}$ & $\begin{array}{l}M \\
\text { and } \\
a_{M}\end{array}$ & $\begin{array}{l}n\left(a_{0}, z, t\right) \\
a_{o}=2-400 \mu \mathrm{m}\end{array}$ & $K_{j}\left(a_{o}, z, t\right)$ & $\xi_{j}\left(a_{o}, z, t\right)$ & $\int_{0}^{z} \xi_{j}\left(a_{o}, z, t\right) M_{j}(z, t) d z$ & $\Re_{\mathrm{j}}$ & $\psi_{\mathrm{j}}$ & 1 & 0 & $\begin{array}{l}\text { Figs. } 9 \text { and } 10 \text { a (mean } \\
\text { proffile) Fig. } 9 \text { e } 10 d, \text { e } \\
\text { (timeseries) Solid circles }\end{array}$ \\
\hline MF2b & $\begin{array}{l}\text { Multi } \\
\text { freq. } \\
0.7 / \\
1.25 / \\
3 \mathrm{MHz}\end{array}$ & $\begin{array}{l}\text { Implicit } \\
\text { iter. Eq. } \\
(8)\end{array}$ & $\begin{array}{l}n(a) \\
\text { Assumed } \\
\text { or bed } \\
\text { sample }\end{array}$ & $\begin{array}{l}M \\
\text { and } \\
a_{M}\end{array}$ & $\begin{array}{l}n\left(a_{o}, z, t\right) \\
a_{o}=2-400 \mu \mathrm{m}\end{array}$ & $K_{j}\left(a_{o}, z, t\right)$ & $\xi_{j}\left(a_{o}, z, t\right)$ & $\int_{0}^{z} \xi_{j}\left(a_{o}, z, t\right) M_{j}(z, t) d z$ & $\Re_{\mathrm{j}}$ & $\psi_{\mathrm{j}}$ & $0.8 \& 1.2$ & 0 & $\begin{array}{l}\text { Figs. } 9 \text { and } 10 \text { a (mean profile) } \\
\text { Fig. } 9 \text { d, e } 10 d \text {, e (timeseries) } \\
\text { Red shaded }\end{array}$ \\
\hline MF2c & $\begin{array}{l}\text { Multi } \\
\text { freq. } \\
0.7 / \\
1.25 / \\
3 \mathrm{MHz}\end{array}$ & $\begin{array}{l}\text { Implicit } \\
\text { iter. Eq. } \\
(8)\end{array}$ & $\begin{array}{l}n(a) \\
\text { Assumed } \\
\text { or bed } \\
\text { sample }\end{array}$ & $\begin{array}{l}M \\
\text { and } \\
a_{M}\end{array}$ & $\begin{array}{l}n\left(a_{o}, z, t\right) \\
a_{o}=2-400 \mu \mathrm{m}\end{array}$ & $K_{j}\left(a_{o}, z, t\right)$ & $\xi_{j}\left(a_{o}, z, t\right)$ & $\int_{0}^{z} \xi_{j}\left(a_{o}, z, t\right) M_{j}(z, t) d z$ & $\Re_{\mathrm{j}}$ & $\psi_{\mathrm{j}}$ & $0.8 \& 1.2$ & 0.1 & $\begin{array}{l}\text { Figs. } 9 \text { and } 10 \text { a (mean profile) } \\
\text { Figs. } 9 d, \text { e, } 10 d, \text { e (timeseries) } \\
\text { Blue shaded Figs. 9b, c, 10b, c } \\
\text { (fields) }\end{array}$ \\
\hline MF3a & $\begin{array}{l}\text { Multi } \\
\text { freq. } \\
0.7 / \\
1.25 / \\
3 \mathrm{MHz}\end{array}$ & $\begin{array}{l}\text { Implicit } \\
\text { iter. Eq. } \\
(7)\end{array}$ & $\begin{array}{l}n(a) \\
\text { Assumed } \\
\text { or bed } \\
\text { sample }\end{array}$ & $M$ & $\begin{array}{r}\bar{a}_{M}(z) \text { from } \\
\text { MF2a }\end{array}$ & $K_{j}\left(\bar{a}_{M}(z)\right)$ & $\xi_{l}\left(\bar{a}_{M}(z)\right)$ & $\int_{0}^{z} \xi\left(\bar{a}_{M}(z)\right) M(z, t) d z$ & $\Re_{\mathrm{j}}$ & $\psi_{\mathrm{j}}$ & 1 & 0 & $\begin{array}{l}\text { Fig. 11a (mean profile) } \\
\text { Fig. 11d \& e (timeseries) Solid } \\
\text { circles }\end{array}$ \\
\hline MF3b & $\begin{array}{l}\text { Multi } \\
\text { freq. } \\
0.7 / \\
1.25 / \\
3 \mathrm{MHz}\end{array}$ & $\begin{array}{l}\text { Implicit } \\
\text { iter. Eq. } \\
(7)\end{array}$ & $\begin{array}{l}n(a) \\
\text { Assumed } \\
\text { or bed } \\
\text { sample }\end{array}$ & $M$ & $\begin{array}{l}\bar{a}_{M}(z) \text { from } \\
\text { MF2c }\end{array}$ & $K_{j}\left(\bar{a}_{M}(z)\right)$ & $\xi_{l}\left(\bar{a}_{M}(z)\right)$ & $\int_{0}^{z} \xi\left(\bar{a}_{M}(z)\right) M(z, t) d z$ & $\Re_{\mathrm{j}}$ & $\psi_{\mathrm{j}}$ & $0.8 \& 1.2$ & 0.1 & $\begin{array}{l}\text { Fig. 11b and c (fields) Grey } \\
\text { shaded }\end{array}$ \\
\hline
\end{tabular}

$\sigma=0.4 a_{o}$. To obtain the acoustic estimates for $\mathrm{a}_{\mathrm{c}}$, in the inversion $a_{M}$ was obtained from values of $a_{o}$ covering the range $2-400 \mu \mathrm{m}$ with a step interval of $\delta a=2 \mu \mathrm{m}$.

In the first multi-frequency case, MF1, no uncertainties were introduced into the forward calculation, $\varepsilon=1$ and $\gamma_{\mathrm{o}}=0$. To simplify the inversion an assumption that sediment attenuation could be ignored, $r \alpha_{s} \ll 1$, led to an explicit solution, further the nearfield correction was ignored, $\psi=1$. In the second case of multi-frequency scenarios, MF2, a more complex implicit iterative inversion was used with $\alpha_{s}$ accounted for and the nearfield correction applied. In MF2 systematic and random errors were added to the forward calculations of the backscattered signal in the evaluation of Eq. (A1.4) and different conditions of uncertainty were examined. As presented in Table 2, in scenarios MF2a, $\varepsilon=1$ and $\gamma_{\mathrm{o}}=0$, in MF2b, $\varepsilon=0.8,1.2$ and $\gamma_{\mathrm{o}}=0$, and in MF2c, $\varepsilon=0.8,1.2$ and $\gamma_{\mathrm{o}}=0.1$. In the third case MF3, the time series profiles of $a_{M}$ from MF2c were averaged to give a temporal mean average profile, $\overline{a_{M}}$, and for the same conditions as MF2a and MF2c the profiles for $M$ were recalculated using $\overline{a_{M}}$. This latter calculation was carried out because the impact of both $\varepsilon$ and $\gamma_{\mathrm{o}}$ on $\overline{a_{M}}$ was relatively weak as will be shown in the following section.

\section{Acoustic estimates for particle size and concentration}

In this section the inversion scenarios described in Section 4 are implemented and the results examined in terms of the accuracy of the acoustic estimates of the suspended particle size and concentration. Having a priori knowledge of the suspension can improve the inversion and using more sophisticated inversions can provide further information on the suspension. For the first three inversion cases SF1, SF2 and SF3, a single frequency ABS was assessed with parameter uncertainties and assumptions made about the suspension as described in Section 4.1 and Table 1. These inversions only calculated profiles of $M$. Following these cases, three cases of multifrequency ABS deployments, MF1, MF2 and MF3, were examined with parameter uncertainties and assumptions made about the suspension as described in Section 4.2 and Table 2. In these inversions profiles of $a_{M}$ and $M$ were obtained. Following the inversion scenarios, further calculations are reported to assess the impact of uncertainties on the profiles of $a_{M}$ and $M$.

5.1. Estimates of suspended sediment concentration, $M$, using single frequency ABS for cases SF1, SF2 and SF3 given in Table 1

The backscatter signal from suspended sediments is a function of the particle size and concentration. If an estimate of the suspended size is available then a single frequency ABS can be used to obtain a measurement of the concentration field. Using the suspension field shown in Fig. 4 the backscattered sound field was computed as described in Section 3.2 using Eq. (A4.1). ABS systems usually operate over the range $0.5-5.0 \mathrm{MHz}$ and for the present simulations the frequency was chosen to be $2.0 \mathrm{MHz}$. From the previous section the bed sediment particle size was given a value of $d_{\mathrm{b} 50}=320 \mu \mathrm{m} \quad\left(a_{o}=a_{\mathrm{bc}}=110 \mu \mathrm{m}\right)$, which gives $x_{o}=0.93$ at $2.0 \mathrm{MHz}$ and places the scattering above the Rayleigh regime $x \ll 1$ and below the geometrical regime $x \gg 1$. Backscattering in the intermediate regime has a lower sensitivity to changes in particle size than in the Rayleigh regime and sediment attenuation is not as significant as it is at higher frequencies; the lower sensitivity to size and attenuation contributes to reducing 
inversion errors. The $2 \mathrm{MHz}$ single frequency $\mathrm{ABS}$ was mounted at $1.0 \mathrm{~m}$ above the bed and had a vertical spatial resolution of $0.005 \mathrm{~m}$.

The conditions for the different cases with their scenarios are presented in Table 1. For the first single frequency case SF1, the simple situation of a single frequency $2.0 \mathrm{MHz}$ ABS deployment was considered. The only information available for the local site was an estimate for $\mathrm{d}_{50}$ of the bed which was $320 \mu \mathrm{m}$. To obtain the suspended concentration the explicit solution of Eq. (4) was used, i.e. $r \alpha_{s} \ll 1, \psi=1$ and it was assumed $\Re$ had previously been measured. As no information was available for $m(a), K_{i}$ was obtained using the intrinsic value for $f_{i} / \sqrt{\rho}$ calculated using the expression in equation (3a) for a particle radius of $a_{o}=d_{50} / 2$. The value for $K_{i}$ was constant over time and height above the bed. The results for this straightforward inversion are shown in Fig. 5. First the inverted computed concentration field, $M$, obtained using backscattered signals without added uncertainties, $\varepsilon=1, \gamma=0$, scenario SF1a, is presented in Fig. 5a. The impact of not accounting for the nearfield is observed between $z=0.9-1.0 \mathrm{~m}$ where the concentration field reduces rapidly. Apart from this nearfield artifact, it can be seen that the temporal structure of the suspension field in Fig. 5a is comparable with that of Fig. 4d, although the magnitude in Fig. 5a is decreasing at a greater rate with $z$.
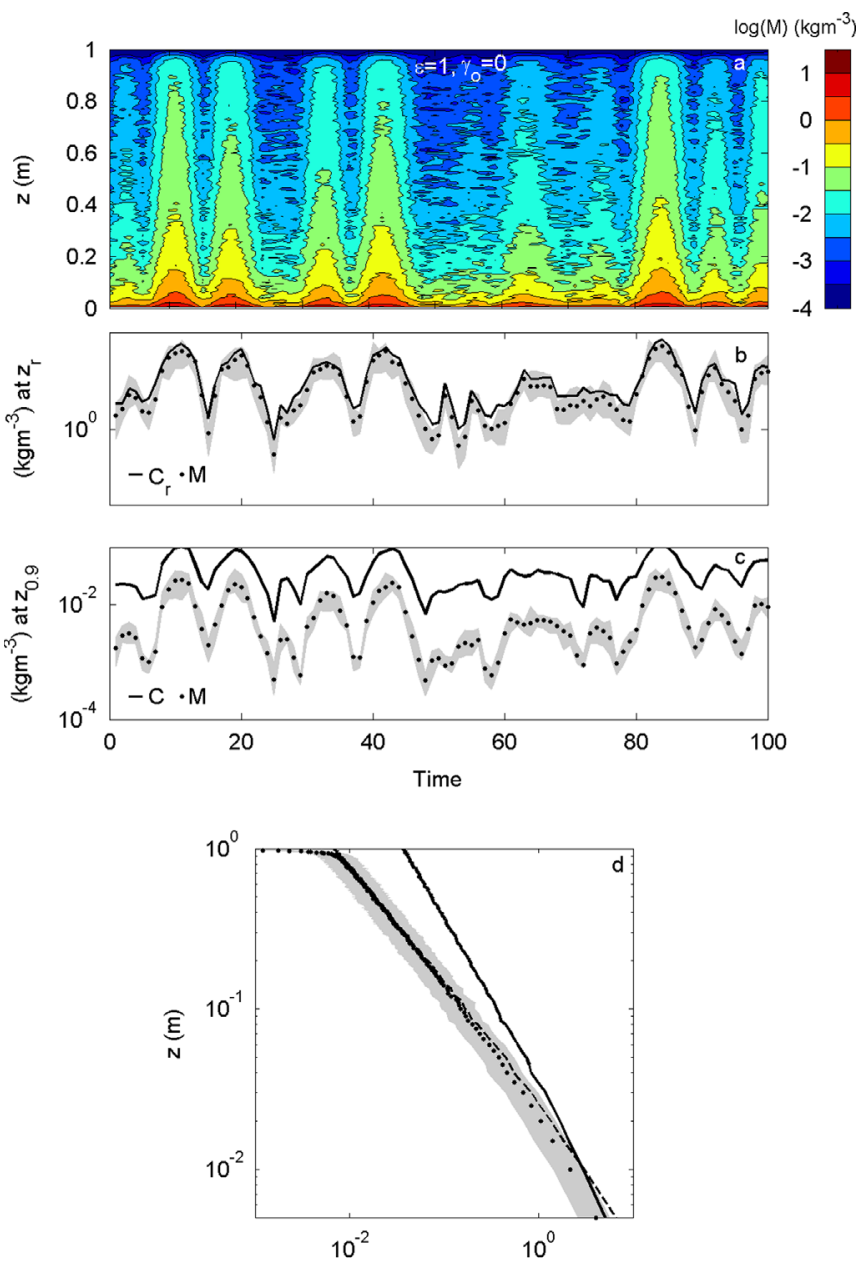

$\left(\mathrm{kgm}^{-3}\right)$

Fig. 5. $2.0 \mathrm{MHz}$ single frequency inversion for $M$ using $a_{o}=d_{\mathrm{b} 50} / 2$ and the intrinsic scattering characteristics, case SF1 in Table 1. (a) Temporal structure of $M$ with height above the bed for $\varepsilon=1$ and $\gamma_{0}=0$. ((b) and (c)) Temporal variation of $C(-)$ and $\mathrm{M}(\bullet)$ respectively at $z_{r}$ and $z=0.9 \mathrm{~m}$, with the grey shaded area representing the uncertainty in $M$ for $\varepsilon=0.8,1.2$ and $\gamma_{0}=0.1$. (d) $\bar{C}(-)$ and $\bar{M}(\bullet)$ with the shaded area representing the uncertainty in $\bar{M}$ and the dashed line with account taken of the sediment attenuation and the nearfield in the calculation for $\bar{M}$.
This is illustrated in Fig. $5 \mathrm{~b}$ and $\mathrm{c}$ in which the concentration from the inversion, $M$, is centered on the solid circles, scenario SF1a, with a shaded area bound by inversions associated with systematic errors $\varepsilon=0.8$ and 1.2 , and random errors $\gamma_{\mathrm{o}}=0.1$ scenario SF1b, and the solid line is from the input concentration field, $C$, at the same value of $z$. The upper boundary of the shaded area is associated with scattering and calibration characteristics that are greater than those assumed for the inversion, i.e. $\varepsilon=1.2$, while the lower boundary is associated with reduced scattering and calibration characteristics than assumed in the inversion, i.e. $\varepsilon=0.8$. The two heights shown are at the reference height $z_{r}=0.005 \mathrm{~m}$ and $z=0.9 \mathrm{~m}$. It can clearly be seen that at $z_{r}, M$ is similar in form and magnitude to $C_{r}$. That the magnitudes are comparable may be somewhat surprising, given $K_{i}$ and $d_{\mathrm{b} 50} / 2$ where used for the inversion, however, coincidentally, $K_{i}=1.43$ for $d_{\mathrm{b} 50} / 2$ and $K=1.73$ for $a_{\mathrm{cb}}$ at $2.0 \mathrm{MHz}$ at $z_{r}$, hence their values are similar and therefore $M$ is a reasonably accurate estimate. At $z=0.9 \mathrm{~m}$ the temporal structure of $M$ is still comparable with $C$, however, the magnitude of $M$ is an order of magnitude smaller. This reduction is due to assuming $a_{o}=d_{50} / 2$ in the inversion, while the actual temporal mean value, $\overline{a_{c}}$, at $0.9 \mathrm{~m}$ was $29 \mu \mathrm{m}$, which has associated lower scattering characteristic. The general trend of increasing departure of $M$ from $C$ with height above the bed is shown by the temporal mean concentration profiles in Fig. 5d. The dashed line in Fig. $5 d$ is an implicit iterative inversion using Eq. (7) in which the nearfield and sediment attenuation were accounted for, scenario SF1c. As can be seen there is only a marginal increase in $\bar{M}$. Therefore using $K_{i}$ and $d_{50} / 2$ in the inversion provides a temporal structure for $M$ which is comparable with $C$, but with $M$ becoming increasingly erroneous in magnitude with $z$. The temporal-spatial mean value for $\overline{M / C}=0.23$; including the nearfield correction and sediment attenuation only increased this value to $\overline{M / C}=0.25$. The suspended concentration field was therefore significantly underestimated for all SF1 scenarios.

In the second single frequency case, SF2, the assumption was made that bed samples from the location site were available. Using the samples the $2.0 \mathrm{MHz}$ ABS could be calibrated for the site specific suspension in the laboratory and $K \Re$ obtained, or if $\Re$ is known, $n(a)$, $a_{o}, f$ and hence $K$ could be estimated from the bed samples using Eqs. (2a), (2c), (3a). Either way the inversion was carried out assuming a suspension with $n_{b}(a)$ (i.e. identical to that of the bed) and invariant with time and height above the bed. The results for such an inversion are shown in Fig. 6 where the same nomenclature as used in Fig. 5 is employed. Fig. 6a, represents the result for scenario SF2a. The results of scanarios SF2a, SF2b and SF2c are shown in Fig. 6b-d. Although expectations may have been for improved agreement between $M$ and $C$, as noted earlier the values for $K$ and $K_{i}$ at $2.0 \mathrm{MHz}$ used in the inversions to obtain Figs. 5 and 6 were within 20\% of one another, with the result that the profiles for $M$ in the two figures are very comparable. In Fig. $6 \mathrm{~b}$ at the high concentration events, when the nearbed suspended sediments have a value of $a_{o}$ equal to that of the bed, the results for $M$ still underestimate $C_{r}$, due to the assumption that $\mathrm{r} \alpha_{s} \ll 1$. Overall the resulting $M$ profiles in Fig. 6 have essentially the same problem as in Fig. 5, the lack of accounting for the particle size sorting with $z$ again results in an increasing underestimate of the suspended concentration with height above the bed.

Owing to the lack of agreement between $M$ and $C$ with $z$ for inversion cases SF1 and SF2 seen in Figs. 5 and 6, in the third case, $\mathrm{SF} 3$, particle size sorting with height above the bed was included in the inversion. Obtaining suspended sediment samples at the deployment site could be used to estimate the sorting, or bed samples could again be used, but combined with an estimate for the temporal mean particle radius profile, $\overline{a_{o}(z)}$, with height above the bed. In the present scenario the latter option was used with $\overline{a_{0}}=a_{b c}=110 \mu \mathrm{m}$ at $z_{r}$ and with a reduction in mean particle size with height above the bed given by Eq. (12b). Therefore using the size profile, $K$ was calculated at each height above the bed and a 

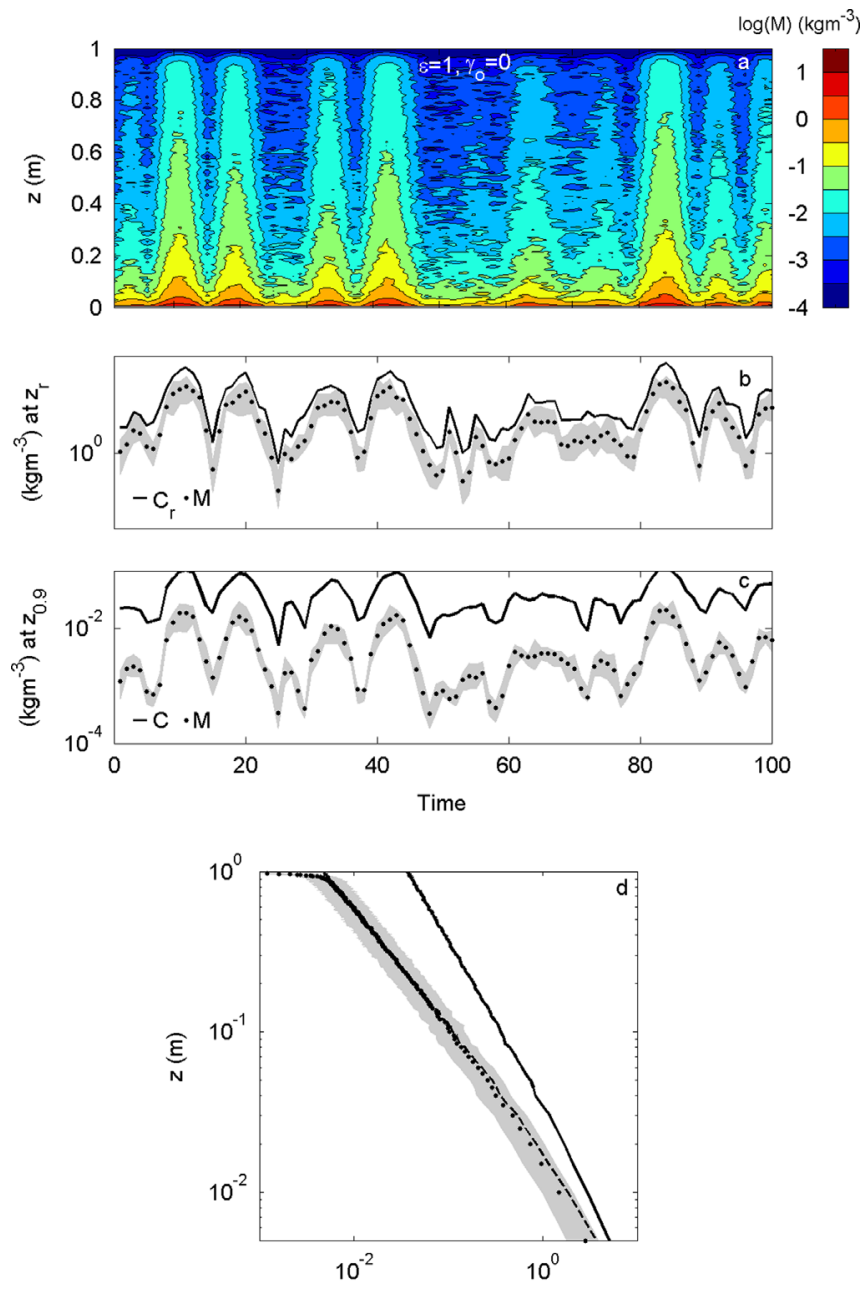

$\left(\mathrm{kgm}^{-3}\right)$

Fig. 6. $2.0 \mathrm{MHz}$ single frequency inversion for $M$ using $a_{o}=a_{\mathrm{bc}}$ and ensemble scattering characteristics, case SF2 in Table 1. (a) Temporal structure of $M$ with height above the bed for $\varepsilon=1$ and $\gamma_{\mathrm{o}}=0$. ((b) and (c)) Temporal variation of $C(-)$ and $M(\bullet)$ respectively at $z_{r}$ and $z=0.9 \mathrm{~m}$, with the grey shaded area representing the uncertainty in $M$ for $\varepsilon=0.8,1.2$ and $\gamma_{\mathrm{o}}=0.1$. (d) $\bar{C}(-)$ and $\bar{M}(\bullet)$ with the shaded area representing the uncertainty in $\bar{M}$ and the dashed line with account taken of the sediment attenuation and the nearfield in the calculation for $\bar{M}$.

time invariant profile for $K$ obtained. The results from this inversion for scenarios SF3a and SF3b are shown in Fig. 7a-c. As would be expected, given the results in Figs. 5 and 6, using a profile size estimate in the inversion results in a substantial improvement in the agreement between $M$ and $C$ with $z$. Both the temporal structure and the magnitude of $M$ agree reasonably well with $C$ at all elevations above the bed, with a temporalspatial mean value, $\overline{M / C}=0.54$, which is a significant improvement over the two previous cases, SF1 and SF2. Again accounting for the nearfield and sediment attenuation in scenario SF3c, shown in Fig. 7d, obtained using Eq. (7) only provides a marginal improvement with $\overline{M / C}$ increasing to 0.58 .

The reason why in SF3c the value of $M$ is still an underestimate is because the particle size profile is based on a reference value at $z_{r}$ of $\overline{a_{0}}=a_{\mathrm{bc}}$ for the bed and this represents the maximum one would expect to obtain as a reference. In practice this value is only achieved for the most dynamic conditions as illustrated in Fig. 4c. Therefore in Fig. 7b and $\mathrm{c}$ the values for $M$ and $C$ are only coincident for the more energetic events and in general $M$ underestimates $C$ because the particle size is overestimated. Furthermore, as shown in Fig. 7b for scenario SF3b, even though the
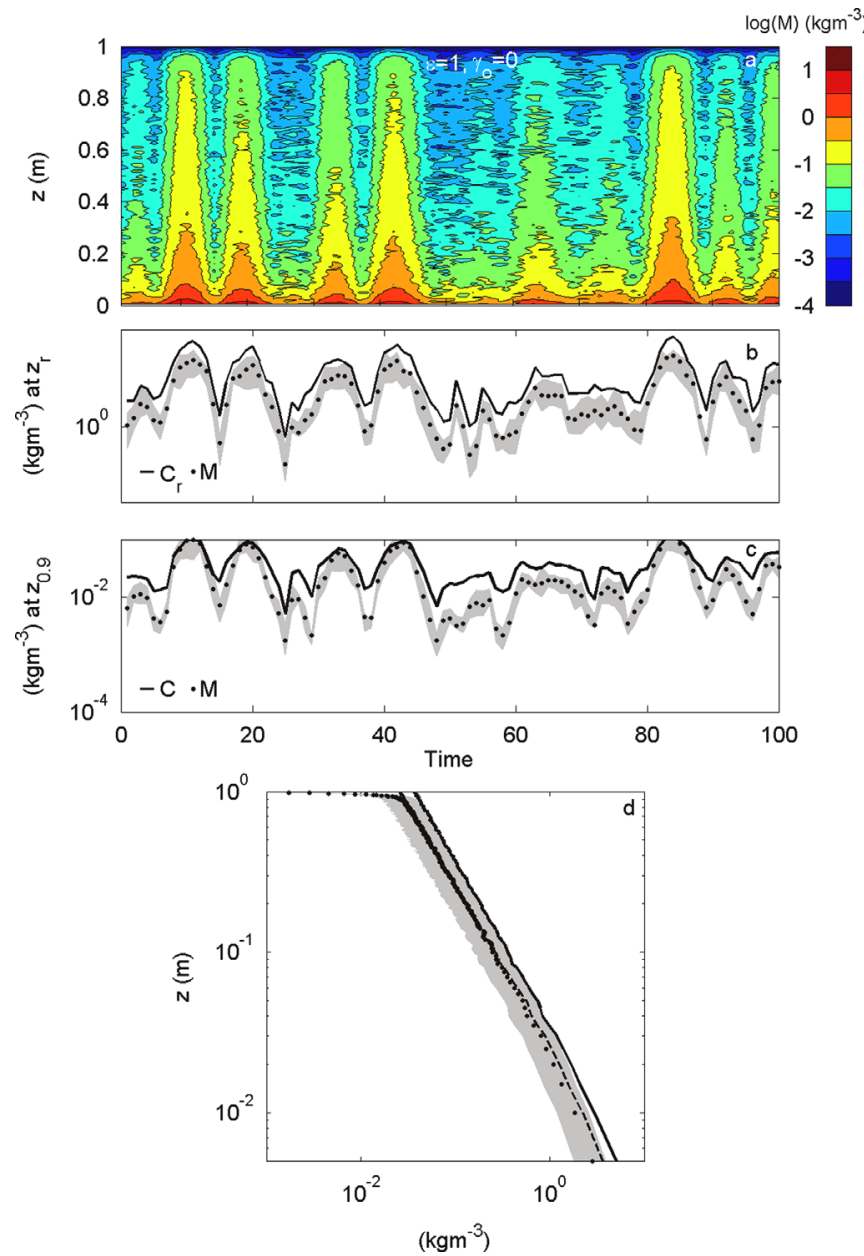

Fig. 7. $2.0 \mathrm{MHz}$ single frequency inversion for $M$ using the profile in Eq. (12b), with $a_{o}=a_{\mathrm{bc}}\left(z / z_{r}\right)^{-1}$ and the ensemble scattering characteristics, case SF3 in Table 1. (a) Temporal structure of $M$ with height above the bed for $\varepsilon=1$ and $\gamma_{0}=0$. (b) and (c) temporal variation of $C(-)$ and $M(\bullet)$ respectively at $z_{r}$ and $z=0.9 \mathrm{~m}$, with the grey shaded area representing the uncertainty in $M$ for $\varepsilon=0.8,1.2$ and $\gamma_{\mathrm{o}}=0.1$. (d) $\bar{C}$ $(-)$ and $\bar{M}(\bullet)$ with the shaded area representing the uncertainty in $\bar{M}$ and the dashed line with account taken of the sediment attenuation and the nearfield in the calculation for $\bar{M}$.

correct value for $a_{o}$ is being employed at $z_{r}$ for the more energetic events, the use of $r \alpha_{s} \ll 1$ results in the concentration during these events being underestimated. Therefore the inclusion of a representative size profile provides a significant improvement in the acoustic estimate of the suspended concentration profile, however, because the size profile is invariant with time and based on the particle size distribution of the bed, it still fails to capture the exact nature of the suspended concentration field. To do this an inversion needs to be carried out which contains information on the time variability of the suspended particle size field. One way of doing this is to use multi-frequency acoustics to also measure particle size.

\subsection{Estimates of suspended sediment particle size, $a_{M}$, and} concentration, M, using multi-frequency ABS for cases MF1, MF2 and MF3 given in Table 2

If particle size as well as concentration is desired, then multiple frequencies are employed and the differential scattering characteristics with frequency of the sediment in suspension is utilised to calculate profiles of the time varying particle size and concentration. In the present study inversions were carried over the range $a_{o}=2-400 \mu \mathrm{m}$ with a step interval of $2 \mu \mathrm{m}$ and $a_{M}$ and $M$ obtained 
at the minimum value of $\Phi$ as expressed by Eq. (8). This range of radii covers the Wentworth grain size scale from fine silt through to coarse sand. A simple approach would be to add only one acoustic frequency to the single frequency system described above, however, the lack of uniqueness in the value of $K$ with particle size makes the inversion problematic; with erroneous results readily generated; this is illustrated in Appendix C. Most publications using multi-frequency ABS have therefore opted for a three frequency system (Crawford and Hay, 1993; Thorne and Hardcastle, 1997; Thosteson and Hanes, 1998; Villard et al., 2000; Smerdon and Caine, 2007, Moore et al., 2013) and it is such a system which is investigated here. Typically ABS have been deployed in sandy environments and operated in the $0.5-5 \mathrm{MHz}$ frequency range. At much lower frequencies scattering is in the Rayleigh region, $x \ll 1$, where backscattering can be weak and at much higher frequencies the combined water and suspended sediment attenuation both reduce the backscattered signal level and introduce instabilities into the inversion (Thorne et al., 1995). With regard to the above, the present study examines scenarios for an ABS operating at $0.7,1.25$ and $3.0 \mathrm{MHz}$ in a sandy environment. As with the $2 \mathrm{MHz}$ single frequency ABS the system was mounted at $1.0 \mathrm{~m}$, above the bed and had a vertical spatial resolution of $0.005 \mathrm{~m}$.

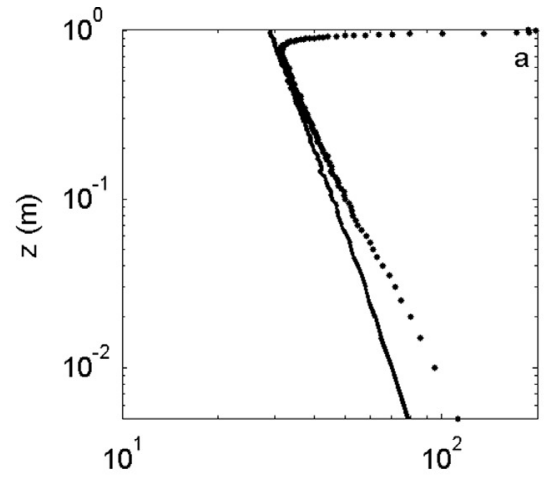

$(\mu \mathrm{m})$

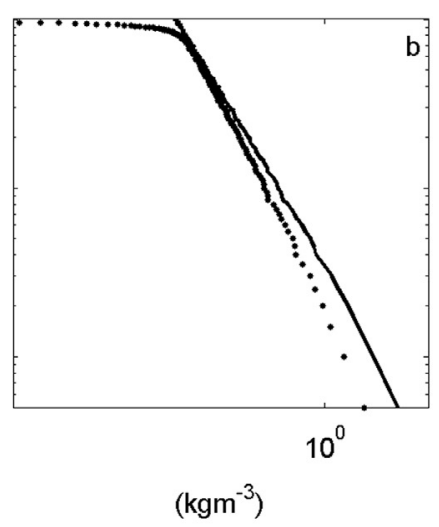

$a_{M}(\mu m)$
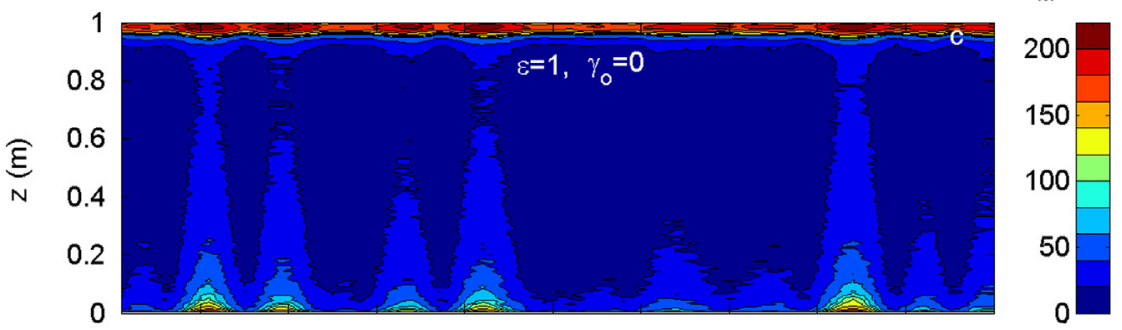

$\log (\mathrm{M})\left(\mathrm{kgm}^{-3}\right)$
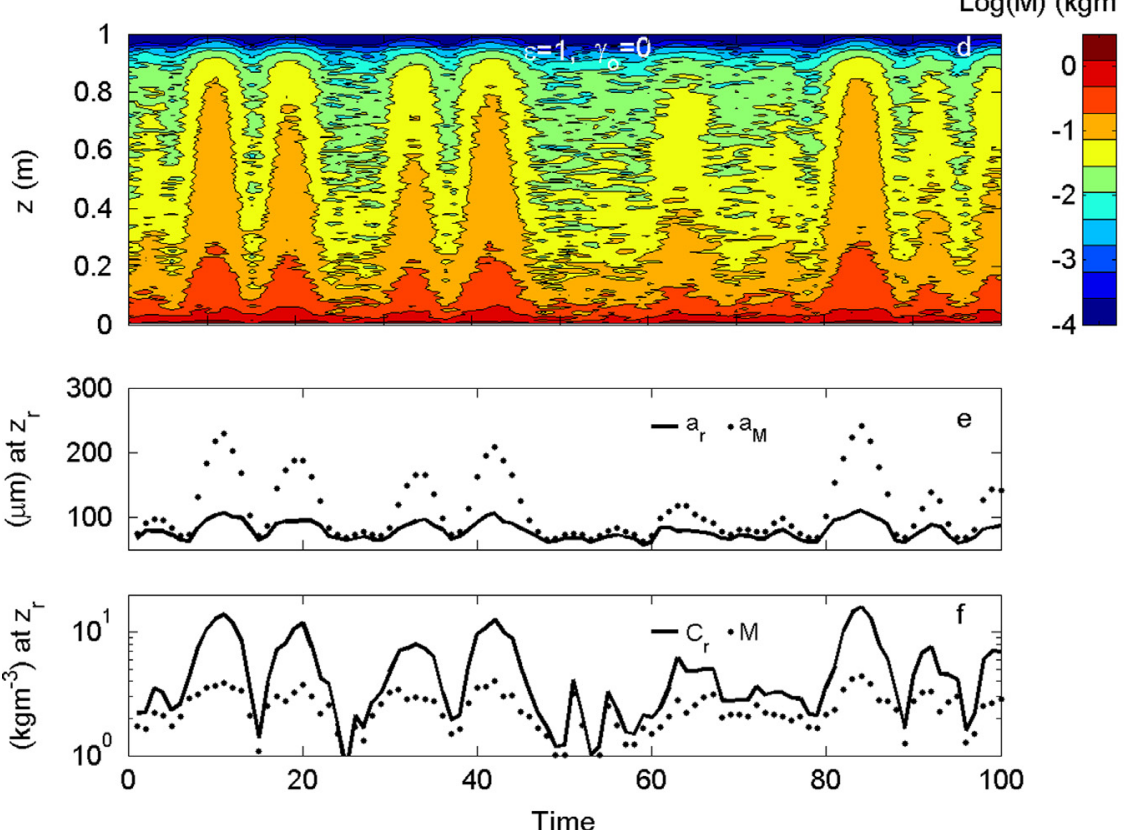

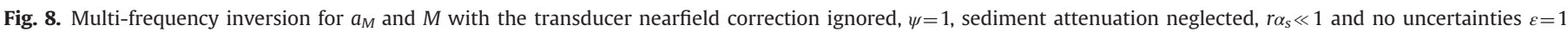

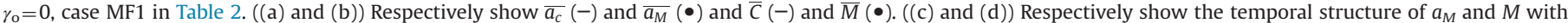
height above the bed. (e) and (f) Respectively show the time series for $a_{r}$ and $a_{M}$ and $C_{r}$ and $M$ at $z_{r}$. 
All the multi-frequency inversions were carried out with a lognormal $n(a)$ having $\sigma=0.4 a_{o}$, which gave them a value for the normalized standard deviation, $\delta$, that was the same as the bed and the $a_{c}$ field. The conditions for the different cases with their scenarios are presented in Table 2. The first inversion for case MF1 yielding $M$ and $a_{M}$ was carried out using Eqs. (1), (2a), (3a) and (8). No uncertainties were introduced into equation (A1.4) for the forward calculation of the backscattered signal, $\varepsilon=1$ and $\gamma_{\mathrm{o}}=0$. For the inversion $\alpha_{s}$ was assumed negligible and no nearfield correction applied, therefore any deviations between the suspension field, $C$ and $a_{c}$, and the acoustic estimates, $M$ and $a_{M}$, was solely due to the simplification of assuming $r \alpha_{s} \ll 1$ and $\psi=1$. Table 2, case MF1, provides an overview of the inversion condition with the outcome from this inversion being shown in Fig. 8. As seen in the mean temporal profiles shown in Fig. 8a and b, the impact of ignoring the nearfield for each transducer produces a local effect close to the $\mathrm{ABS}$, with the particle size over estimated and the concentration underestimated. Between approximately $z=0.2-0.8 \mathrm{~m}$ the mean profiles of $a_{M}$ and $M$ from the ABS are consistent with the suspension field. However, below $z=0.2 \mathrm{~m}$ the ABS inversion increasingly overestimates the particle size and underestimates the concentration. As can be seen in Fig. $8 \mathrm{c}$ and $\mathrm{d}$ the general temporal-spatial form of the suspension field is retained in the inversion, although, as shown in Fig. $8 \mathrm{e}$ and $\mathrm{f}$, close to the bed during the higher suspension events, the lack of accounting for sediment attenuation in the inversion, leads to significant overestimates of particle size and underestimates of concentration. Overestimates of $a_{r}$ by a factor of two and underestimates of $C_{r}$ by a factor of five occurred during the most dynamic suspension events. Therefore neglecting attenuation, when using a multi-frequency ABS, to extract particle size and concentration, can be problematic and this is particularly acute at the more interesting near bed higher concentration events.

In the second multi-frequency case, MF2, the forward calculation for the backscattered signal had uncertainty conditions of
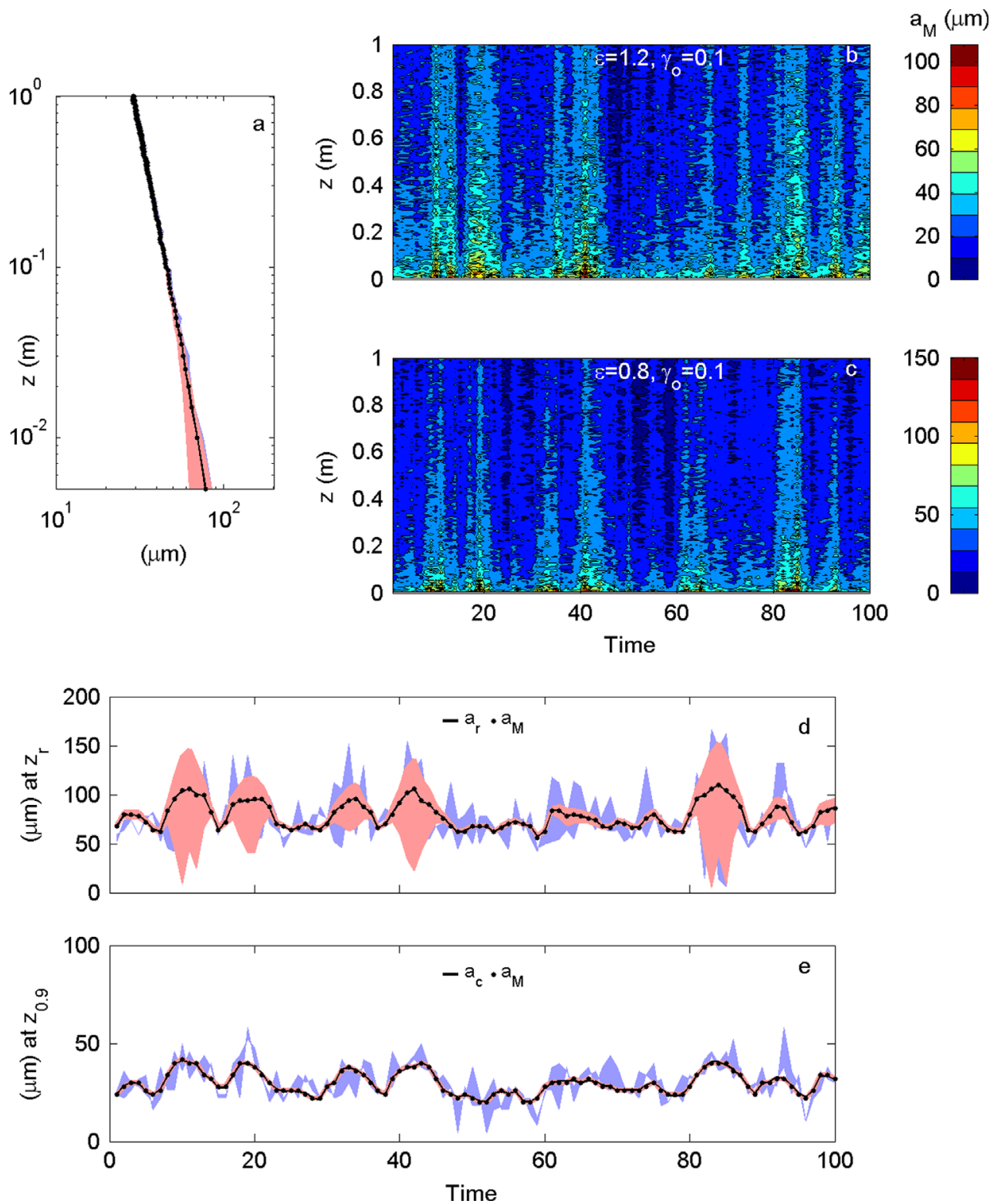

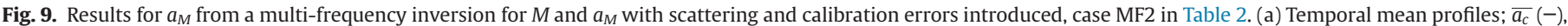

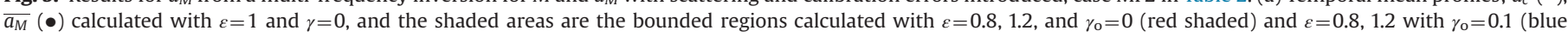

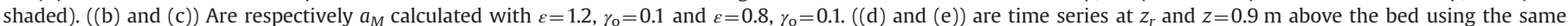
nomenclature as (a). 
$\varepsilon=1.0$ and $\gamma_{\mathrm{o}}=0$, MF2a, $\varepsilon=0.8,1.2$ and $\gamma_{\mathrm{o}}=0$, MF2b, and $\varepsilon=0.8$, 1.2 and $\gamma_{\mathrm{o}}=0.1$, MF2c; again these scenarios are summarised in Table 2. For this case a full inversion using Eqs. (1)-(3), (6)-(8) was employed with account taken of $\alpha_{s}$ and $\psi$. Figs. 9 and 10 respectively show the results for the acoustic estimates of particle size, $a_{M}$, and concentration, $M$.

In Fig. 9a, the time averaged particle radius profiles, $\overline{a_{M}}$, with regions of uncertainty and $\overline{a_{c}}$ are shown. The shaded red and blue regions show the envelopes of the bounded inversion results for the uncertainty conditions MF2b and MF2c, respectively. The solid line is the $\overline{a_{c}}$ profile and the solid circles the $\overline{a_{M}}$, profile for MF2a. The temporal averaged results from the inversions show that $\overline{a_{M}}$ is consistent in form to $\overline{a_{c}}$ with $z$, with the region of uncertainty increasing as the height above the bed is reduced. In Fig. 9a the two regions of uncertainty (red and blue) overlapped considerable. In Fig. $9 \mathrm{~b}$ and $\mathrm{c}$ are respectively shown $a_{M}$ for $\varepsilon=1.2, \gamma_{\mathrm{o}}=0.1$ and $\varepsilon=0.8, \gamma_{\mathrm{o}}=0.1$, correspond to inversion scenarios MF2c in Table 2 . As can be seen in the figures the broad temporal structure of $a_{M}$ is comparable to that shown in Fig. 4a for $a_{c}$, although somewhat noisier owing to the introduction of random errors into the backscatter calculation. Analysis of the inversion shows that close to the bed for $\varepsilon>1, a_{M}<a_{c}$ and for $\varepsilon<1, a_{M}>a_{c}$, as illustrated by the colour bar scales in Fig. 9b and c. In Fig. 9d and e the temporal variability is highlighted more clearly at the reference height and at $z=0.9 \mathrm{~m}$. The nomenclature for these figures is the same as for Fig. 9a; the solid line is $a_{c}$, the solid circles MF2a and the red and blue shaded areas are respectively MF2b and MF2c. Fig. 9d shows the results at $z_{r}$ for MF2b, $\gamma_{\mathrm{o}}=0$, red shaded area, and good estimates for $a_{M}$ were obtained when the concentrations were relatively low, however, at the higher concentrations the region of uncertainty is large. This is due to positive feedback arising from the implicit iterative inversion when sediment attenuation becomes significant (Thorne et al., 2011b) and errors propagate and increase with range from the transducer. For the scenario where when $\gamma_{\mathrm{o}}=0.1$, blue shaded area, MF2c, the introduction of a random component uncorrelated at each frequency, introduces further variability into the estimate of $a_{M}$ which can be significant. In Fig. 9e for the scenario MF2b, $\gamma_{\mathrm{o}}=0$ at $z=0.9 \mathrm{~m}$, corresponding to the red shaded area, the time series for $a_{M}$ is almost identical to that of $a_{c}$. This arises due to the manner in which $a_{M}$ was obtained
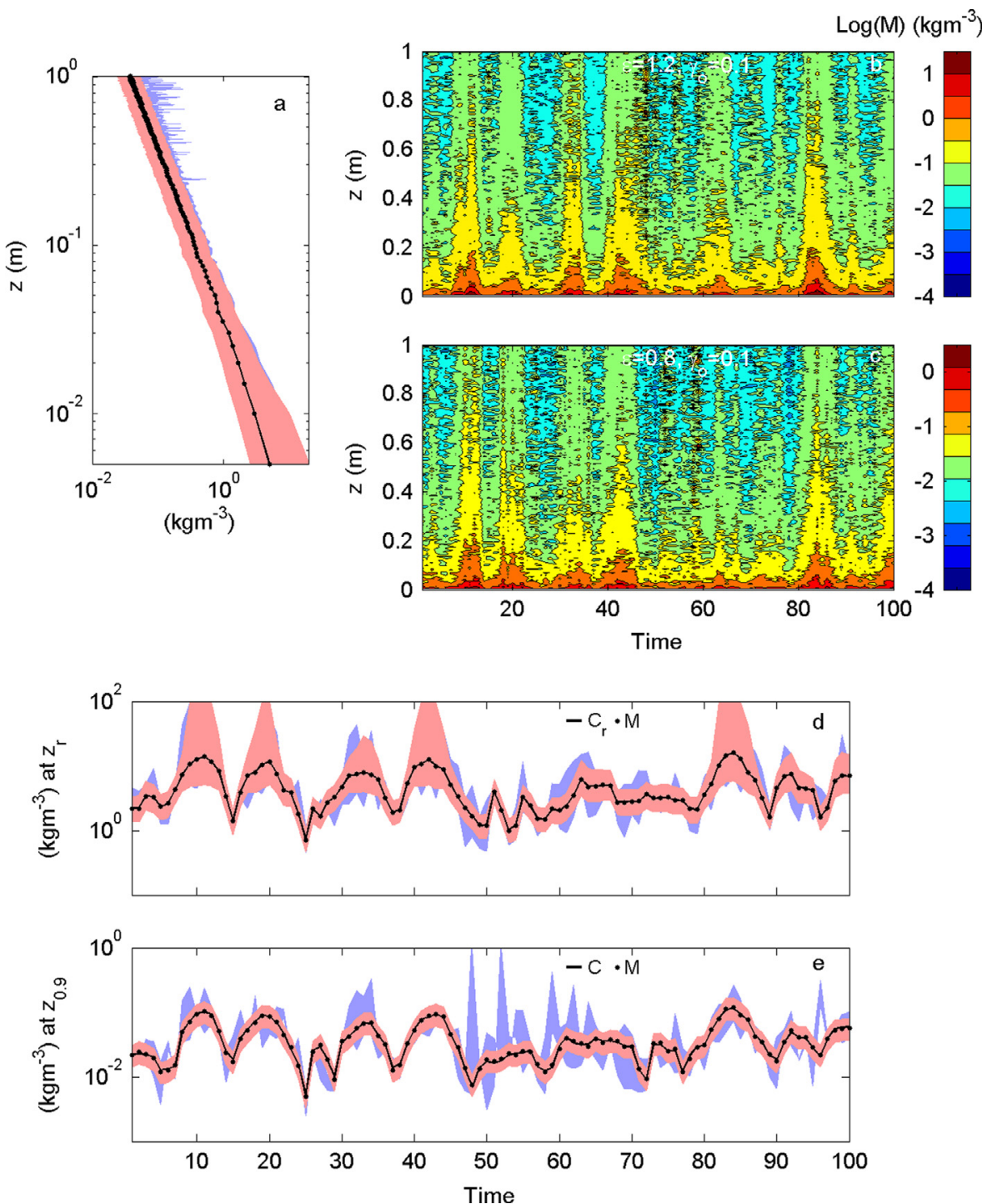

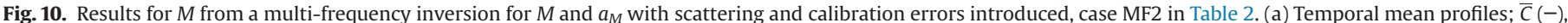

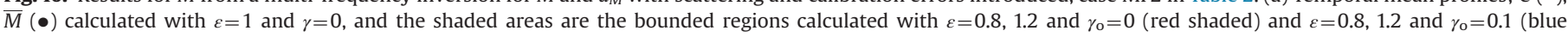

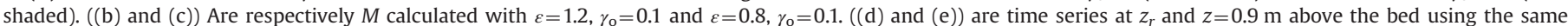
nomenclature as (a) 
from the minimum of $\Phi$ using Eq. (8), where changes in $\varepsilon$ have a similar impact on $\sigma_{\mathrm{m}}\left(a_{o}\right)$ and $M\left(a_{o}\right)$ when sediment attenuation is not significant. Therefore, there is only a marginal impact on $\Phi$ and hence the values of $a_{M}$. For the scenario MF2c, when $\gamma_{0}=0.1$, corresponding to the blue shaded area in Fig. 9e, the gross underlying structure of the time series is present, however, the introduction of a random component uncorrelated at each frequency, introduces variability into the temporal estimate of $a_{M}$ which can be significant.

In Fig. 10 the inversion output for $M$ is shown using the same nomenclature as Fig. 9 and for the same case MF2. In Fig. 10a, the time averaged concentration profile, $\bar{M}$, with regions of uncertainty corresponding to scenarios MF2a, MF2b and MF2c are presented with $\bar{C}$. As with $\overline{a_{M}}$ and $a_{c}$, it can be seen that the results for $\bar{M}$ are similar in form to $\bar{C}$ with height above the bed and with the region of uncertainty increasing towards the bed, although this region of uncertainty is significantly greater than for the case of $\overline{a_{M}}$. In Fig. 10b and $\mathrm{c}$ are respectively shown $M$ for $\varepsilon=1.2, \gamma_{\mathrm{o}}=0.1$ and $\varepsilon=0.8, \gamma_{\mathrm{o}}=0.1$, corresponding to inversion scenario MF2c. In the figures it can be observed that the general pattern in the suspended sediment concentration is broadly consistent with those of $C$ shown in Fig. 4d. Results from the inversion shows that when $\varepsilon>1, M>C$ and when $\varepsilon<1, M<C$, as indicated by the colour bar scales in Fig. 10b and c. In Fig. 10d is shown the time series of $M$ at $z_{r}$. For the scenario MF2b, red shaded area, the periods of lower concentration yield values of $M$ comparable to $C_{r}$, however, at the higher concentration events the values for $M$ are substantially overestimated for $\varepsilon=1.2$ and underestimated for $\varepsilon=0.8$. As with $a_{M}$, these substantial uncertainties just above the bed at high concentration events are due to the multi-frequency coupled errors propagating with range when attenuation becomes significant. For the MF2c scenario, blue shaded area, the addition of the random noise component generates further uncertainties. In 10e is shown the time series for $M$ at $0.9 \mathrm{~m}$ above the bed, for the case MF2b when $\gamma_{\mathrm{o}}=0$, the region of uncertainty in $M$ closely follows $C$, with $M$ marginally overestimating $C$ when $\varepsilon=1.2$ and underestimating when $\varepsilon=0.8$. The addition of the random noise component, MF2c, with $\gamma_{\mathrm{o}}=0.1$, can add significant variability to $M$.

The fluctuations in $a_{M}$ associated with the random component, $\gamma_{\mathrm{o}}$, has a significant impact on the temporally estimated values of $M$. However the random component reduces towards zero with
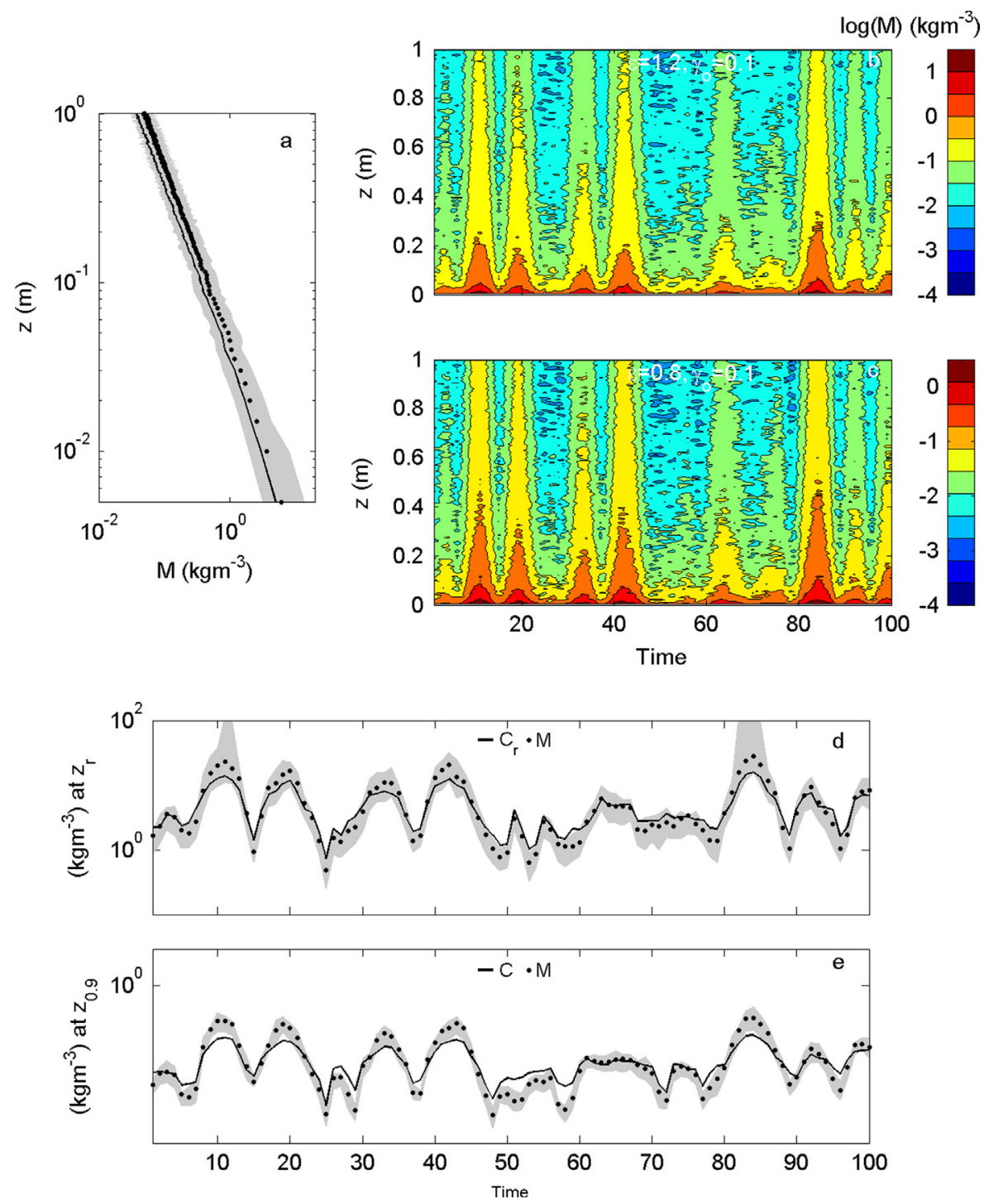

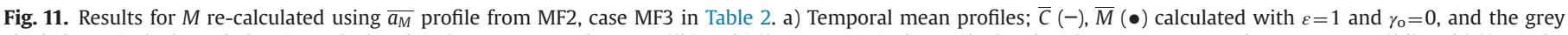

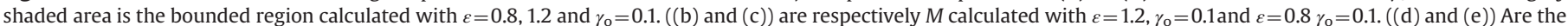
time series at $z_{r}$ and $z=0.9 \mathrm{~m}$ above the bed using the same nomenclature as (a). 
temporal averaging. Therefore if a lower temporal resolution for $a_{M}$ is acceptable improved estimates for $M$ can be obtained. Using the three $\overline{a_{M}}$ profiles shown in Fig. 9a, obtained from the MF2a, $\varepsilon=1$ and $\gamma_{\mathrm{o}}=0$, and MF2c, $\varepsilon=0.8, \gamma_{\mathrm{o}}=0.1$ and $1.2, \gamma_{\mathrm{o}}=0.1$ scenarios, $M$ was re-calculated for scenario MF3a and MF3b. This resulted in the new values for $M$ shown in Fig. 11. The mean profile of $M$ for $\varepsilon=1$ and $\gamma_{\mathrm{o}}=0$, MF3a, is given by the solid circles, with the region of uncertainty, represented by the grey shaded area bounded by $\varepsilon=0.8,1.2$ and $\gamma_{\mathrm{o}}=0.1, \mathrm{MF} 3 \mathrm{~b}$, is shown in Fig. 11a and is seen to be comparable with Fig. 10a. The temporal structure of $M$ shown in Fig. 11b and c respectively for $\varepsilon=1.2$ and $\varepsilon=0.8$ each with $\gamma_{\mathrm{o}}=0.1$, are somewhat less noisy than Fig. 10b and c, due to the use of $\overline{a_{M}}$, and more consistent with Fig. 4d. However, it is in the detailed times series illustrated by the results at $z_{r}$ and $z=0.9 \mathrm{~m}$, respectively, in Fig. 11d and e that show clear improvements over Fig. 10 in the values and uncertainties in $M$. Therefore by reducing the variability in $a_{M}$ due to random uncertainties, through forming a temporally averaged profile, $\overline{a_{M}}$, from the output from a multifrequency iterative implicit inversion, it is possible to reduce uncertainties and improve the accuracy of the high temporalspatial re-calculated values of $M$. However, because a mean profile for $a_{M}$ is being utilized, it is not possible to fully capture the exact variation in $C$, as illustrated in Fig. 11d and e. Although not presented for brevity, similar results to those shown in Fig. 11 were also obtained using multi-frequency temporally averaged $\overline{V_{m}^{2}}$ to obtain $\overline{a_{M}}$ and again using $\overline{a_{M}}$ for the high spatial-temporal inversion for $M$.

\subsection{Sensitivity analysis and error estimations as a function of uncertainty}

The inversion outcomes in Figs. 9-11 provide a specific illustration of the impact on $a_{M}$ and $M$ of systematic and random variability in the backscattered signal. To obtain a broader assessment of the impact of uncertainties the backscattered signal was calculated over a range of values for $\varepsilon$ and $\gamma_{\mathrm{o}}$ and full iterative implicit inversions carried out accounting for $\alpha_{s}$ and $\psi$ using Eqs. (1)-(3), (6)-(8). The suspension field in Fig. 4 was again used and backscatter signals calculated as; (i) the systematic error $\varepsilon$, increased from 0.5 to 1.5 with $\gamma_{\mathrm{o}}=0$ and (ii) the range of variation of the independent random errors increased as $\gamma_{\mathrm{o}}$ changed from 0.0 to 0.2 with $\varepsilon=1$. The first case in Fig. 12a shows the temporalspatial mean values for $\overline{a_{M} / a_{c}}$ and $\overline{M / C}$ with error bars derived from the standard deviation of the ratios. The results show that $\overline{a_{M} / a_{c}} \approx 1$ and as previously mentioned this arises due to changes in $\varepsilon$ having a similar impact on $\sigma_{\mathrm{M}}\left(a_{o}\right)$ and $M\left(a_{o}\right)$ and hence $\Phi$ and therefore only marginally impacting on the values of $a_{M}$. The values for $\overline{M / C}$ are somewhat different and shows that when the scattering and calibration characteristics are lower than assumed in the inversion, $\varepsilon<1$, the value for $M$ significantly underestimates $C$ and if $\varepsilon>1$ then $M$ is substantially greater than $C$. This is consistent with the observations made for the SF cases when $a_{o}$ was assumed and only $M$ was being estimated. In general, however, it is unlikely that uncertainties would apply exactly equally at each frequency, therefore the second case looks at the impact of random variability; this is shown in Fig. 12b and c. In Fig. 12b it can be observed that $\overline{a_{M} / a_{c}}$ marginally increased with $\gamma_{\mathrm{o}}$, however, the standard deviation steadily increased reaching a standard deviation of around $\pm 50 \%$ for $\gamma=0.2$. In Fig. $12 \mathrm{c}$ the results for $\overline{M / C}$ are shown by the solid circles and the form is quite different to that of $\overline{a_{M} / a_{c}}$ with both $\overline{M / C}$ and the error bars increasing substantially above $\gamma_{\mathrm{o}} \approx 0.05$. This substantial increase is associated with the dependence of $M$ on $a_{o}$ through $K$. As illustrated in Fig. 1, when $x<1$, the dependency of $M$ on $a_{o}$ tends to $a_{0}^{3}$, therefore errors in the realizations of $a_{M}$ which reduce estimated particle size have a substantial impact on the computed a

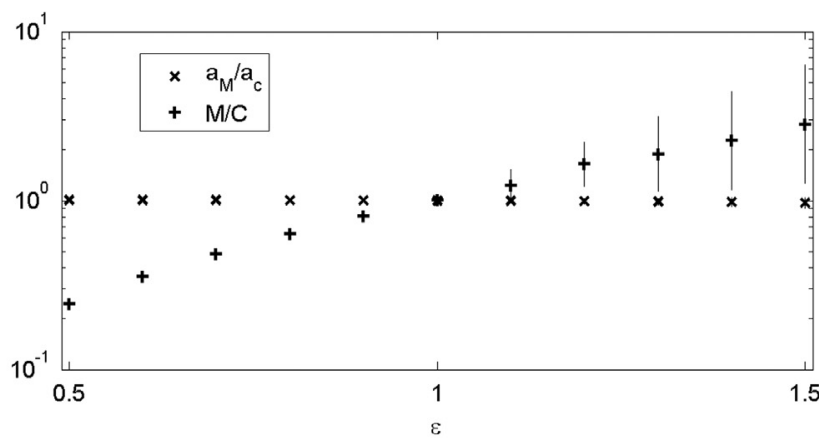

b

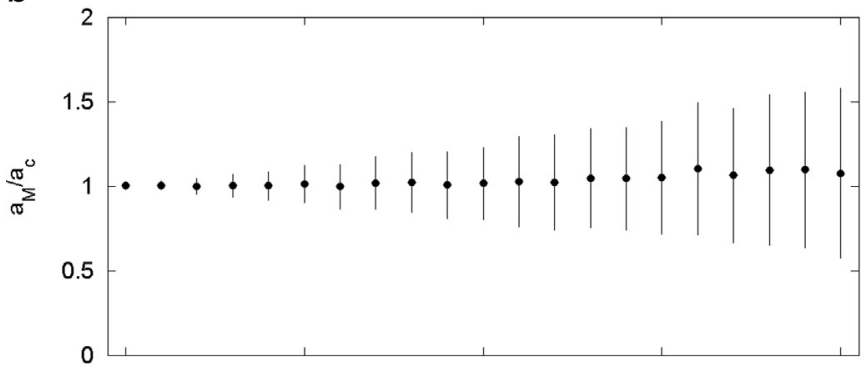

C

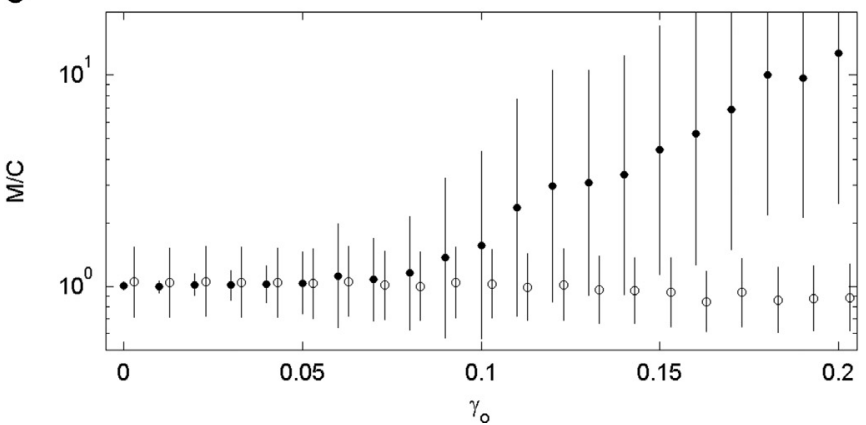

Fig. 12. (a) Calculation for the temporal-spatial averages for $\overline{a_{M} / a_{c}}(x)$ and $\overline{M / C}$ $(+)$ with error bars for variations in $\varepsilon$. ((b) and (c)) Respectively show the variation in $\overline{a_{M} / a_{c}}(\bullet)$ and $\overline{M / C}(\bullet)$ with error bars for increasing $\gamma_{\mathrm{o}}$. Also shown in (c) is the result for $\overline{M / C}$ (o) using the $\overline{a_{M}}$ profile for the calculation of $M$.

variability of $M$. Also when realizations result in increases in $a_{M}$, then owing to the dependency of $M$ on $a_{o}$ being weaker for $x \geq 1$, there is a resulting bias in $\overline{M / C}$ leading to values increasingly becoming greater than unity as $\gamma_{o}$ increases. This impact varies with mean particle size (Moate and Thorne, 2009), decreasing as mean particle size increases as shown in Appendix D. If the data used to obtain Fig. 12b, is employed to provide $\overline{a_{M}}$ profiles for each value of $\gamma_{\mathrm{o}}$, and the inversions repeated using $\overline{a_{M}}$ then the results shown by the open circles in Fig. 12c are obtained. The impact of the uncertainties is substantially reduced with $\overline{M / C}$ remaining close to unity and error bars being approximately constant. Fig. 12 illustrates in broad terms the impact on the calculated values for $a_{M}$ and $M$ that variations in the backscattered signal have on the output of the inversion. For accurate inversions of $a_{M}$ and $M$, precise knowledge of $n(a), K, \xi$ and $\Re$ are required and increasing so as particle size reduces, although results for $M$ can be significantly improved using $\overline{a_{M}}$ in the calculation due to the nominally unbiased estimates of $\overline{a_{M}}$.

\subsection{Relationship between the $a_{M}$ and $d_{50}$}

It was remarked in Section 3.1, that the acoustic size, obtained from inverting the backscattered signal, is not the median mass 


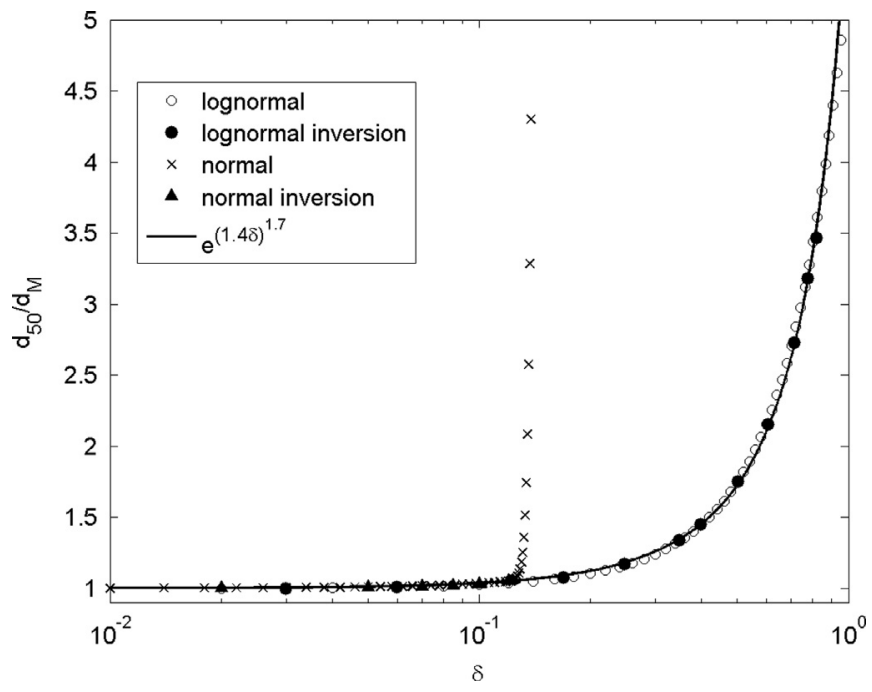

Fig. 13. Evaluation of the ratio of the median mass diameter to the acoustic mean diameter, $d_{50} / d_{M}$, with the mass size normalised standard deviation, $\delta=\sigma_{\mathrm{m}} / a_{\mathrm{m}}$, assuming $n(a) \propto m(a) / a^{3},(\mathrm{o}, \mathrm{X})$, and from a full acoustic inversion, $(\bullet, \mathbf{\wedge})$, using profiles $\bar{C}$ and $\overline{a_{c}}$ for lognormal and normal probability density functions for $m(a)$. The solid line represents a fit to the lognormal distribution data.

diameter, $d_{50}$, used almost universally in sediment transport models to define the size of the sediments in suspension. The acoustic measurement of particle diameter, $d_{M}=2 a_{M}$, derived using a range of $a_{o}$, given by Eq. (2c), is an estimate for $2 a_{c}$ and is based on a probability density function representing the number of particles with size, $n(a)$, rather than the mass of the particles with size, $m(a)$. If the assumption is made that the particles are nominally spherical in shape then $n(a)$ is obtained from $m(a) / a^{3}$. Using this relationship the variation of $d_{50} / d_{M}$, with the normalised standard deviation, $\delta=\sigma_{\mathrm{m}} / a_{\mathrm{m}}$, can be calculated for lognormal and normal mass distributions. The results are shown in Fig. 13, with the open circles and crosses respectively representing the value for $d_{M}$ calculated using $m(a) / a^{3}$ and the solid circles and solid triangle representing $d_{M}$ calculated using a full acoustic iterative implicit inversion of the backscattered signal with Eqs. (1)-(3), (6)-(8) using forward scattered fields based on $\overline{a_{c}}$, and $\bar{C}$. For the lognormal mass distribution it can readily be observed that as $\delta$ increases the value for $d_{50} / d_{M}$ accordingly becomes larger and is moderately well represented by an expression of the form.

$d_{50}=d_{M} e^{(1.4 \delta)^{1.7}}$

This relationship allows $d_{50}$ to be readily obtained from the acoustically measured value of $d_{M}$. For the case of a normal mass distribution the same expression is applicable for $\delta \leq 0.1$, however, above this value $d_{50} / d_{M}$ steeply rises due to the rapid increase in the small particles in the $n(a)$ distribution derived from the $m(a)$. Hence the small size tail of a normal mass distribution can have a significant effect on the acoustically estimated size and therefore care must be taken when applying a normal distribution fit to measured mass size distributions to ensure the fine fraction of the suspended sediment are actually of this form. In general, if sediment samples are available, it may be more prudent to convert the measured mass in each size range to a particle number distribution and then apply the measured $n(a)$ in Eq. (2). It would then be necessary to convert the acoustically measure $d_{M}$ to $d_{50}$ by forming a curve similar to that presented in Fig. 13 to calculate $d_{50}$.

\section{Discussion and conclusion}

As commercial instruments have become available the application of acoustic backscatter systems, ABS, to the measurement of particle size and concentration has steadily increased in use over the past two decades. The majority of users of ABS have a limited background in acoustics and therefore obtaining suspended sediment parameters from $A B S$ data can on occasions prove to be somewhat problematic. The aim of the present work has therefore been to provide an overview and assessment, of the methodology commonly used to extract suspended sediment parameters, from backscattered acoustic data collected over mobile beds of inorganic non-cohesive sediments at normally employed low megahertz ABS frequencies.

The focus of the study has been on the inversion of ABS data to obtain suspension profiles, with a précis on the forward problem for calculating the backscattered signal in Appendix A. Eqs. (1)(3), (6)-(8) provide the general solution for estimating suspended sediment concentration profiles; this solution is an implicit expression in $M$, it is solved iteratively and progressively with range along the direction of sound propagation, and requires a methodology to simultaneous obtain $a_{M}$ if particle is size unknown. This solution can be considerably simplified if sediment attenuation can be ignored, $r \alpha_{s} \ll 1$, an estimate for the suspended particle size is available, and interest is in the transducer farfield. This resulted in Eq. (4), where after accounting for $\alpha_{w}, M$ at any range is simply proportional to $V_{m}^{2}$. This solution has the benefit of simplicity, requiring only a single frequency $A B S$. However, it is restricted to the farfield, the assumption $r \alpha_{s} \ll 1$ and particle size is invariant with height and time above the bed. The restriction of only transducer farfield scattering can readily be addressed using the nearfield expression of Downing et al (1995) and $\alpha_{s}$ can be evaluated using Eq. (2b) and applying the iterative formulations given in Eqs. (6) and (7). This inversion therefore still only requires a single frequency $\mathrm{ABS}$ and the inversion is reasonably straight forward, although it is subject to a constant particle size with $z$ and time. If the requirement is to obtain time series profiles of $a_{M}$ and $M$, then multi-frequency $\mathrm{ABS}$ is required and the systems adopted generally use three frequencies. The use of three rather than two frequencies helps to stabilise the inversion. The extraction of $a_{M}$ from multi-frequency backscatter data increases the complexity and computation time of the acoustic inversions as described in Eq. (8) and the inversion becomes increasingly time consuming when $\alpha_{s}$ is significant. Further to carry out the inversion for $a_{M}$ an estimate for $n(a)$ is required, since, as show in Fig. 1, the suspension scattering characteristic are impacted by having a range of particle sizes in suspension. The extraction of $a_{M}$ and $M$ from a multi-frequency ABS therefore requires a greater level of understanding of the interaction of sound with suspended sediments than when only $M$ is required.

The backscattered signal, $V_{m}^{2}$, used in the inversion, to calculate $M$ or $M$ and $a_{M}$, requires the calculation of an average value from an ensemble of independent backscattered signals. This arises because of the statistical nature of the scattered signal which leads to $\sigma_{\mathrm{e}}\left(V_{m}^{2}\right) / V_{m}^{2}=1 / \sqrt{\eta}$, where $\eta$ is the number of independent samples. In the present study the reduction of the autocorrelation function of the backscattered signal has been used to assess the independence of samples and this led to a time interval of around $5 \mathrm{~ms}$ for independent samples. The $5 \mathrm{~ms}$ time interval provided a nominal value for independent samples, although this will vary depending on frequency and the intensity of the flow turbulence. In rough turbulent flows, the root-mean-square turbulent horizontal velocity, $\sqrt{\overline{u^{\prime 2}}}$, is around $15-20 \%$ of the mean velocity, $u_{o}$, (Heathershaw, 1976) and decorrelation can be considered to occur when the relative movement of the particles is greater than $\lambda / 4$, 
therefore an estimate for $\tau_{o}$ may be $\tau_{o} \approx \lambda / u_{o}$. The limitation on the time interval between independent samples leads to a tradeoff between spatial-temporal resolution and the accuracy of the estimated values for $a_{M}$ and $M$.

To investigate the acoustic estimates of $a_{M}$ and $M$ a suspension field was generated. The field was constructed so that it had a vertical time-averaged sediment concentration profile corresponding to the well-known Rouse profile. In the absence of a general theory for vertical size sorting, the imposed vertical sediment size formulation was chosen on the basis of previous observations obtained by Thorne et al., 2011a over rippled beds. Although in practice the validity of this mean size sorting formulation is not guaranteed and vertical temporal differential settling velocities were not rigorously specified, the simulated suspension field offered the opportunity to assess inversions when size sorting effects are present.

Using the representative suspension field, the forward problem of propagating sound through the suspension was computed and the backscattered signal obtained. Different backscattered signals were calculated to account for uncertainties in; (i) the system calibration, (ii) the formulated scattering characteristics and (iii) the scattering statistics. This variability in the backscattered signal provided the opportunity to assess the impact of uncertainty on the calculated profiles of $a_{M}$ and $M$. To examine the estimated values for $a_{M}$ and $M$ a number of scenarios were considered, in the first instance only $M$ was obtained from the inversion and this was followed by $a_{M}$ and M. Three cases SF1, SF2 and SF3, were considered for the inversion of $M$ based on a single frequency 2.0 MHz ABS. The outcome from these inversions were presented in Figs. 5-7 and show that the temporal structure of $M$ remained consistent with $C$, however, the values for $M$ reduced with height at a greater rate than $C$, owing to the lack of accounting for size sorting. The main factor for improving agreement between $M$ and $C$ in these scenarios was to obtain an estimate for the variation in $a_{c}$ with height above the bed and use this in the inversions.

Following on from the $2.0 \mathrm{MHz}$ studies three cases, MF1, MF2 and MF3, were carried out for calculating $a_{M}$ and $M$ using a multifrequency $\mathrm{ABS}$ inversion at 0.7, 1.25 and $3.0 \mathrm{MHz}$. The first focused on an inversion neglecting $\alpha_{s}$ and setting $\psi=1$. The results in Fig. 8 showed that using $\psi=1$ introduced an artifact near the transducers, however, the more important consequence was that during periods of high concentration, the assumption that $\mathrm{r} \alpha_{s} \ll 1$, resulted in a significant overestimate of particle size and underestimate of concentration in the bottom $0.1 \mathrm{~m}$ above the bed. The results of introducing uncertainties into the inversion for $a_{M}$ and $M$ were illustrated in Figs. 9 and 10. These showed that for no random errors, $\gamma_{\mathrm{o}}=0, a_{M}$ and $M$ provided reasonable estimates for $a_{c}$ and $C$ for the lower concentration events and not to close to the bed. However, for the higher concentration events and close to the bed, when attenuation was significant, the positive feedback in the implicit iterative formulation led to large errors in the estimated values for $a_{c}$ and $C$. Introduction of a random component with $\gamma_{o}=0.1$, caused further uncertainties which could be significant. To reduce uncertainties in the estimate of $M$ it was shown in Fig. 11 that using a time average profile for $a_{M}$ in the inversion could improve results for $M$.

To more generally assess the impact of uncertainties, $a_{M} / a_{c}$ and $M / C$ were evaluated over a wide range of uncertainty levels, for $\varepsilon=0.5-1.5$ with $\gamma_{\mathrm{o}}=0$ and for $\gamma_{\mathrm{o}}=0-0.2$ for $\varepsilon=1$. For the first case $\overline{a_{M} / a_{c}} \approx 1$ while $\overline{M / C}$ increasingly diverged from unity. In the second case $\overline{a_{M} / a_{c}} \approx 1$, although the standard deviation increased with $\gamma_{\mathrm{o}}$ while for $\overline{M / C}$ both the mean estimate and standard deviation increased substantially with $\gamma_{\mathrm{o}}$. For the case when profiles of $\overline{a_{M}}$ were used and only high temporal-spatial values of $M$ were being estimated, the mean value for $\overline{M / C}$ remained close to unity as $\gamma_{\mathrm{o}}$ increased and the region of uncertainty was substantially reduced.

The value for $a_{M}$ obtained from the acoustic inversion is not the size most sedimentologists use to categorise the suspension. Sedimentologists use the median mass diameter, $d_{50}$, and it is $d_{50}$ which is used in most sediment transport formulae. The relationship between $a_{M}$ and $d_{50}$ depends on the probability density function of $m(a)$, and depending on the distribution estimated for $n(a)$ obtained from fitting $m(a)$ to a measured mass size distribution, the results for the relationship can be problematic. The more prudent approach would be to assume the particles were nominally spherical and convert each size fraction of the measured mass distribution into a number size distribution, formulate $n(a)$, and either fit an analytic distribution to $n(a)$ or use the actual measured distribution. In either case, $n(a)$ will need to be related to $m(a)$ to convert the acoustic measurement of $a_{M}$ to the usually accepted size of $d_{50}$ used in most sediment transport formulations.

Finally this article has aimed at providing an overview of the application of acoustics to the measurement of suspended sediment profiles over inorganic non-cohesive beds. The theoretical background, statistics, and errors have been examined through a limited number of scenarios. It is not possible to cover all situations which will be encountered in the field, but this work does aim to provide some guidance as to what may be expected especially when vertical size sorting is present. From the results obtained here, it is suggested that it would be judicious not only to carry out inversions with best estimates of system calibration and suspension scattering characteristics, but to also adjust these values within the bounds of expected uncertainties to ascertain the impact on the acoustic estimates of $a_{M}$ and $M$.

\section{Acknowledgements}

The study was supported by the European Commission Sixth Framework Programme through the Integrating Activity HYDRALAB III, SANDS, Contract no. 022441, the European Community's Seventh Framework Programme through the Integrating Activity HYDRALAB IV, WISE, Contract no. 261520 and NERC, UK project COHBED. PDT would like to thank the OSUG department of the University Joseph Fourier of Grenoble, France, for the funded sabbatical Professorship in 2011 which initiated this work.

\section{Appendix A. The forward problem; calculating the backscattered signal}

Here a précis of the acoustic backscattering from an aqueous suspension is outlined. The amplitude of the backscattered signal, $\mathrm{V}$, from a particle in the beam of an ABS using a disc transceiver can be expressed as (Sheng and Hay, 1988; Thorne and Hanes, 2002)

$V=\frac{a f_{i} V_{T} T_{s} R_{s} g_{R} D^{2}(\theta) e^{-2 r \alpha_{w}}}{2 r^{2}}$

$a$ is the particle radius, $f_{i}$ is the intrinsic backscatter form function (Vogt and Neubauer, 1976), $V_{T}$ is the transmit voltage, $T_{S}$ is the transmit sensitivity, $R_{S}$ is the receive sensitivity, $g_{R}$ is the voltage gain of the receiver, $D(\theta)$ is the transceiver directivity function, $\theta$ is the angle the scatter subtends to the acoustic axis, $r$ is the range to the particle and $\alpha_{w}$ is the attenuation due to water absorption. For incoherent scattering from an elemental volume, $\delta \mathrm{v}$, with $N$ particles per unit volume, the elemental mean-square backscattered signal is (Clay and Medwin, 1997)

$\delta V_{m}^{2}=\left\{V^{2}\right\} N \delta v$ 
where \{\} represents an average over a number of independent backscatter signals squared, $V^{2}$. For a circular transceiver the elemental volume can be written as $\delta v=r^{2} \sin \theta d \theta d \phi d r$ allowing $V_{m}^{2}$ to be expressed as

$V_{m}^{2}=f^{2}\left(V_{T} T_{S} R_{s} g_{R}\right)^{2} \frac{3 M}{16 \pi \rho<a>} \int_{r-r_{o} / 4}^{r+r_{o} / 4} \int_{0}^{\pi / 2} \int_{0}^{2 \pi} \frac{e^{-4 r \alpha}}{r^{2}} D^{4} \sin \theta d \phi d \theta d r$

$N$ has been replaced by the mass concentration, $M, \rho$ is the particle density, $r_{o}$ is the transmitted pulse length and $\left.f^{2}=\langle a\rangle<a^{2} f_{i}^{2} / \rho\right\rangle\left|<a^{3}\right\rangle$, where $<>$ represents an average over the particle size distribution, $n(a)$. The integrals can be readily evaluated (Hay, 1991; Thorne and Hardcastle, 1997) resulting in

$V_{m}^{2}=\left(\frac{K \Re}{r \psi}\right)^{2} M e^{-4\left(r \alpha_{w}+\alpha_{s}\right)}$

where

$$
\begin{aligned}
& \psi=\frac{1+1.35\left(r / r_{n}\right)+\left(2.5\left(r / r_{n}\right)\right)^{3.2}}{1.35\left(r / r_{n}\right)+\left(2.5\left(r / r_{n}\right)\right)^{3.2}} \\
& K=\left(\frac{f^{2}}{<a>}\right)^{1 / 2} ; \quad \alpha_{s}=\int_{0}^{r} \xi M d r ; \quad \xi=\frac{3 \chi}{4<a>} ; \\
& \Re=V_{T} T_{s} R_{s} g_{R}\left(\frac{3 \tau}{16}\right)^{0.5} \frac{0.96}{k A_{t}}
\end{aligned}
$$

a

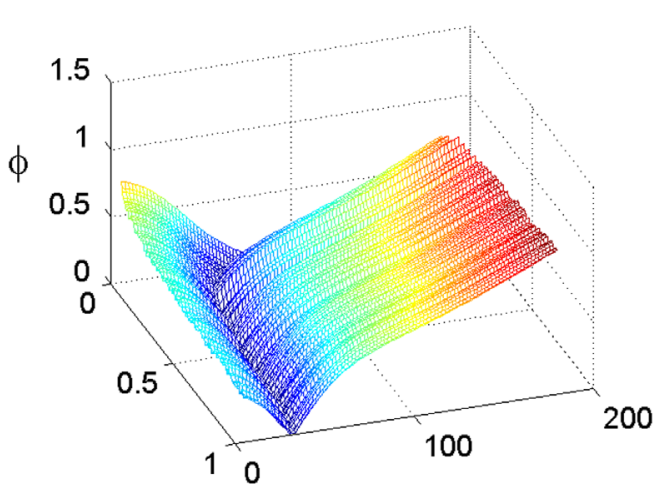

d

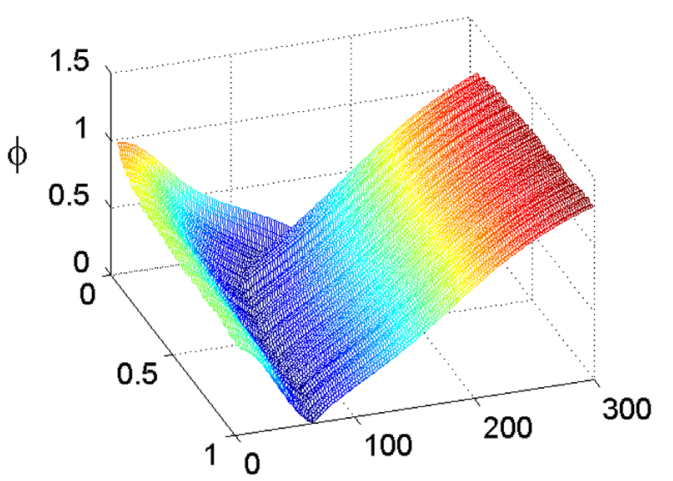

g

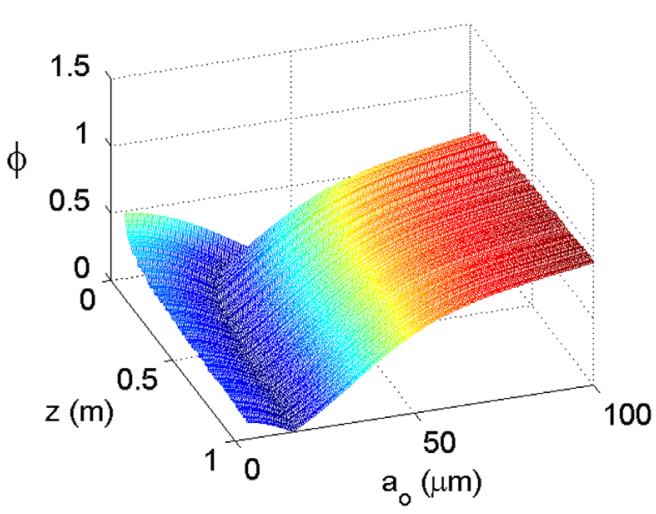

b

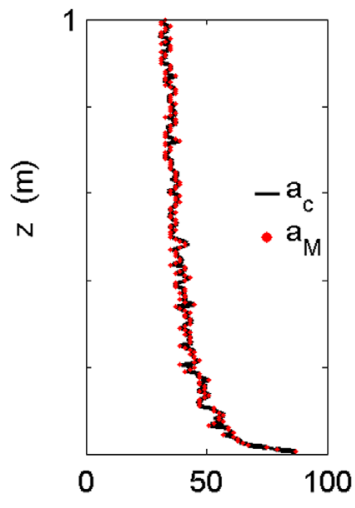

C

e

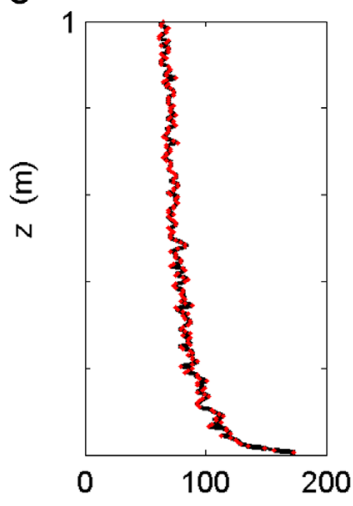

f
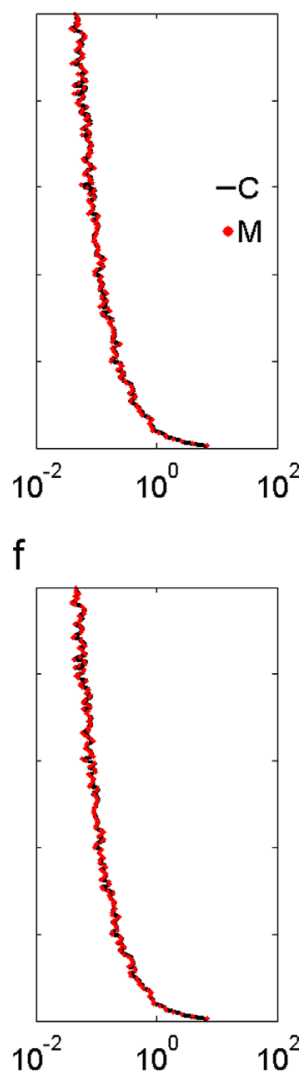

h

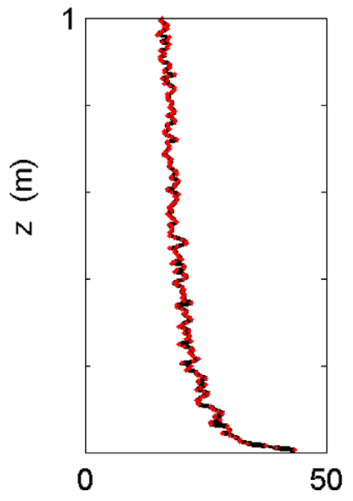

$(\mu \mathrm{m})$ i

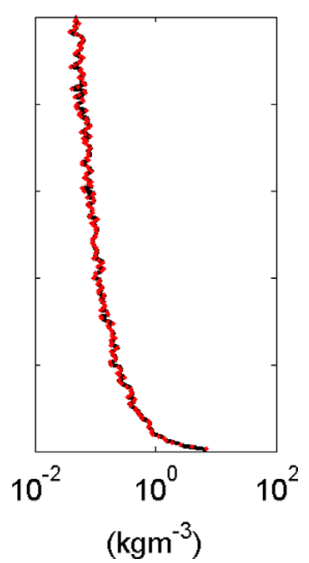

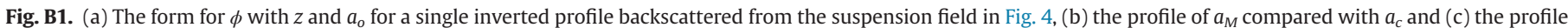
of $M$ compared with $C$. ((d)-(f)) Repeat calculations with $2 a_{c}$ and ((g)-(i)) with $a_{c} / 2$. 
$\alpha_{s}$ is the sediment attenuation, $\psi$ is a transducer nearfield correction factor (Downing et al., 1995), $r_{n}=\pi A_{t}^{2} / \lambda$ is the transducer nearfield, $A_{t}$ the transducer radius, $\left.\chi=<a><a^{2} \chi_{i}|\rho>|<a^{3}\right\rangle, \chi_{i}$ is the intrinsic normalised total backscattering cross-section (Thorne et al., 1993; Schaafsma and Hay, 1997; Moate and Thorne, 2013), and $k$ is the wavenumber of the sound in water. Eq. (A1.4) represents the forward scattering problem; if the composition of the suspension is known the backscattered signal can be calculated. It is the backscattered signal calculated by Eq. (A1.4), with systematic, $\varepsilon$, and random $\gamma_{\mathrm{o}}$ errors introduced, using the suspension field given in Fig. 4 with the $n(a)$ in Fig. 3 that is used in the inversion scenarios. In the present study the focus is on the rearrangement of Eq. (A1.4) making $M$ the dependent variable, as presented in Eq. (1), and estimating $M$ and $a_{M}=a_{o}(<a>)$ through inversion methodologies.

\section{Appendix B. Methodology for estimating $a_{M}$ and $M$.}

Here the procedure for extracting particle size is illustrated. To obtain particle size and concentration from the acoustic inversion requires a methodology to calculate size. A number of approaches have been adopted (Hay and Sheng, 1992; Crawford and Hay, 1993; Thorne and Hardcastle, 1997; Thosteson and Hanes, 1998; Thorne et al., 2007). These approaches have been examined and that of Thorne et al., 2007 was found to be at least as robust as any of the approaches and generally better. However, the approach is computationally intensive, with Eqs. (1)-(3), (6)-(8) being solved for a range of $a_{o}$, at each of the three frequencies, 0.75 , 1.25 and $3.0 \mathrm{MHz}$, and sequentially along the backscattered profile. Eq. (8) outlines the procedure where a solution for $a_{o}$ is sought by minimizing $\Phi=\sigma_{M}\left(a_{o}\right) / M\left(a_{0}\right)$, where $\sigma_{M}$ is the standard deviation and $M$ the mean mass, obtained from the three frequencies, for each value of $a_{o}$, over a prescribed range of $a_{o}$, sequentially at each range bin.

To illustrate the particle size inversion process, one single backscatter profile from the suspension field shown in Fig. 4, has been inverted using Eqs. (1)-(3), (6)-(8) with $n(a)$, for $a_{o}=$ $2-400 \mu \mathrm{m}$ with a step interval of $\delta a_{o}=2 \mu \mathrm{m}$ and the result for $\Phi$ is shown in Fig. B1a between 1 and $200 \mu \mathrm{m}$ for clarity. For the calculation $\varepsilon=1, \gamma=0$, the nearfield correction was applied and account was taken of sediment attenuation. The figure clearly shows a minimum occurring in the surface of $\Phi$ with $\mathrm{z}$ and $a_{o}$. The locus of the minima for $a_{o}$ yields $a_{M}$ which is plotted in Fig. B1b with height above the bed, $z$, and compared with the $a_{c}$ profile. As can be observed, within the step interval of $\delta a_{0}$, the value for $a_{M}$ is in agreement with $a_{c}$. In Fig. B1c the profile of $M$, associated with the minimum value of $\Phi$ is compare with $C$ and good agreement is observed. The same process has been repeated in Fig. B1d-f for a suspension field having twice the particle size and in Fig. B1g-i for a suspension field having half the particle size. As can be seen the inversion was successful in all three cases.

\section{Appendix C. Using two or three frequency ABS inversion}

When a three frequency ABS is deployed three backscattered signal are collected. These can be used as frequency pairs to obtain three estimates of $a_{M}$ and $M$ by minimizing $\Phi$ for each pair. Alternatively all three frequencies can be combined and $\Phi$ minimised to give a single estimate for $a_{M}$ and $M$. To examine each approach $\overline{a_{c}}$ and $\bar{c}$ were used and backscatter signals calculated as systematic and random errors were both varied independently at each frequency over values between $\varepsilon=0.975-1.025$ and $\gamma_{0}=0.025$ and 100 backscattered realization were computed. A full inversion was carried out using Eqs. (1)-(3), (6)-(8) with the nearfield correction applied and account taken of sediment attenuation.
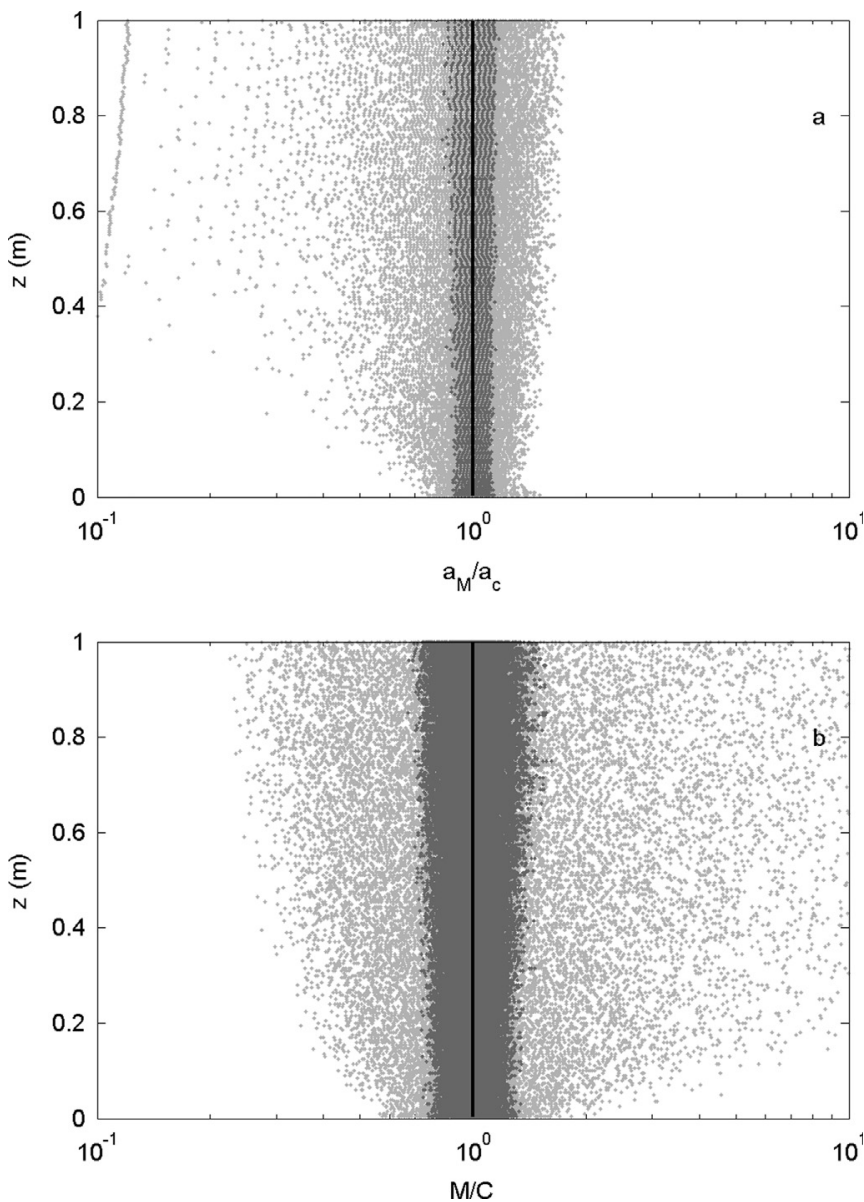

Fig. C1. Using $\overline{a_{c}}$ and $\bar{C}$ profiles, (a) $a_{M} / \overline{a_{c}}$ and (b) $M / \bar{C}$, were calculated using dual frequency pair inversions, light grey profiles, and triple frequency inversions, dark grey profiles, for 100 backscattered realization of $\overline{a_{M}}$ and $\bar{M}$ for values of $\varepsilon$ between $0.975-1.025$ and $\gamma_{\mathrm{o}}=0.025$.

The 100 profiles of $a_{M}$ and $M$ were respectively normalised by $\overline{a_{c}}$ and $\bar{C}$. The results are shown in Fig. $C 1$, the outcome from the three frequency pairs is indicated by the light grey data points and from the three frequency combination by the darker grey points, the solid lines are $a_{M} / a_{c}$ and $M / C$ for $\varepsilon=1, \gamma_{0}=0$. It can be clearly seen that the impact of errors on the two frequency inversions is significantly greater than for the three frequency inversion. The three frequency inversion reduces the degree of ambiguity in $a_{M}$, due to the form of $K$, and adds stability by further constraining the inversion.

\section{Appendix D. Impact of particle size on inversion uncertainties}

To assess the impact of particle size on the accuracy and uncertainty of $a_{M}$ and $M$, the inversions carried out to provide the data for Fig. 12 were repeated using the same $C$ field but with particle radii of $1.5 a_{c}$ and $a_{c} / 1.5$. The results are presented in Fig. D1a-c for $1.5 a_{c}$ and D1d-f for $a_{c} / 1.5$ using the same nomenclature as Fig. 12 and they show that when the particle size increased $\overline{M / C}$ accuracy improved and uncertainties decreased, while when particle size reduced accuracy decreased and uncertainties increased. The difference with particle size is due to the dependence of $M$ on $a_{o}$; when $x<1$ the dependency of $M$ on $a_{o}$ tends to $a_{0}^{3}$, which increases the variability and bias of $\overline{M / C}$, while increases in $a_{o}$ lead to a weaker dependency of $M$ on $a_{o}$ for $x \geq 1$, resulting in reduced uncertainty and bias in $\overline{M / C}$. The result of using 
a

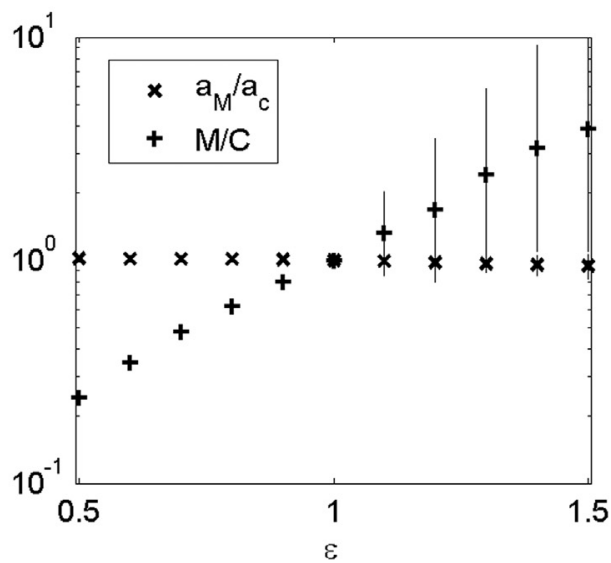

b

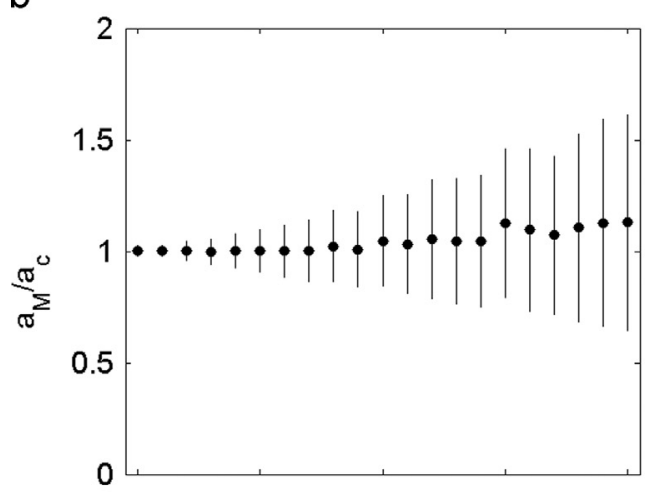

C

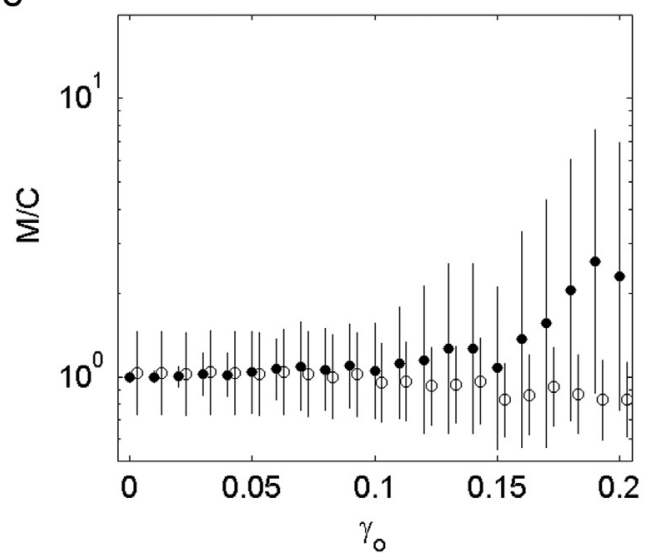

d

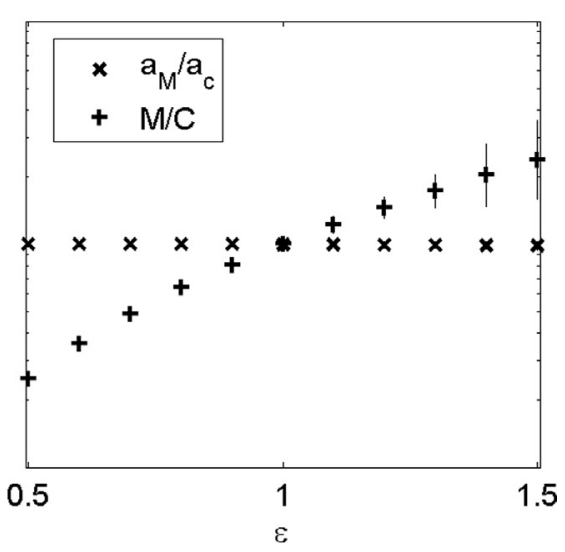

e

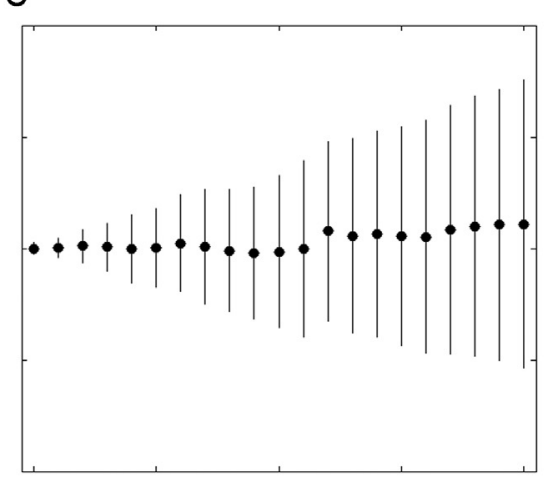

f

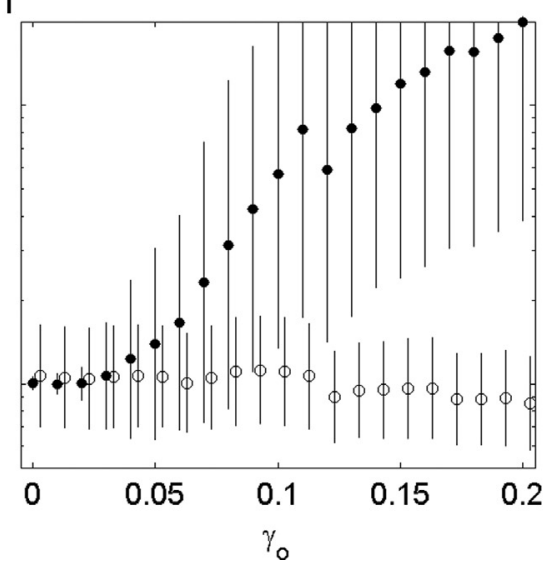

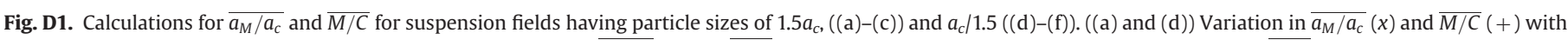

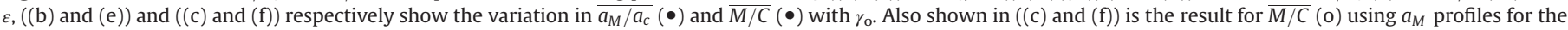
calculation of $M$.

$\overline{a_{m}}$ and re-computing $M$ yielded more accurate and less variable estimates of $M$.

\section{References}

Amoudry, L.O., Souza, A.J., 2011. Deterministic coastal morphological and sediment transport modeling: a review and discussion. Rev. Geophys. 49, RG2002, http: //dx.doi.org/10.1029/2010RG000341.

Betteridge, K.F.E., Thorne, P.D., Cooke, R.D., 2008. Calibrating multi-frequency acoustic backscatter systems for studying near-bed suspended sediment transport processes. Cont. Shelf Res. 28, 227-235.

Bolanos, R., Thorne, P.D., Wolf, J., 2012. Comparison of measurements and models of bed stress, bedforms and suspended sediments under combined currents and waves. Coastal Eng. 62, 19-30.
Clay, C.S., Medwin, H., 1997. Acoustical Oceanography. John Wiley and Sons, Canada.

Crawford, A.M., Hay, A.E., 1993. Determining suspended sand size and concentration from multifrequency acoustic backscatter. J. Acoust. Soc. Am. 94 (6), 3312-3324.

Davies, A.G., Thorne, P.D., 2008. Advances in the study of moving sediments and evolving seabeds. Surv. Geophys. 29 (1), 1-36.

Downing, A., Thorne, P.D., Vincent, C.E., 1995. Backscattering from a suspension in the near field of a piston transducer. J. Acoust. Soc. Am. 97 (3), 1614-1620.

Faran, J.J., 1951. Sound scattering by solid cylinders and spheres. J. Acoust. Soc. Am. 23 (4), 405-418.

Gaunaurd, G.C., Uberall, H., 1983. RST analysis of monostatic and bistatic acoustic echoes from an elastic sphere. J. Acoust. Soc. Am. 73 (1), 1-12.

Gordon, L, 1996, Acoustic Doppler Current Profiler-Principles of Operation: A Practical Primer: RD Instruments, San Diego, CA, pp41 available at 
〈http://www.comm-tec.com/Library/Technical_Papers/RDI/Broadband\%20Primer.pdf

Hay, A.E., 1983. On the remote acoustic detection of suspended sediments at long wavelengths. J. Geophys. Res. 88 (C12), 7525-7542.

Hay, A.E., 1991. Sound scattering from a particle-laden turbulent jet. J. Acoust. Soc. Am. 90, 2055-2074.

Hay, A.E., Sheng, J., 1992. Vertical profiles of suspended sand concentration and size from multifrequency acoustic backscatter. J. Geophys. Res. 97 (C10), $15661-15677$.

Hay, A.E., Bowen, A.J., 1994. Coherence scales of wave-induced suspended sand concentration fluctuations. J. Geophys. Res. 99 (C6), 12749-12765, http://dx.doi. org/10.1029/94JC00290.

Hay, A.E., 2011. Geometric bottom roughness and the bed state storm cycle. J. Geophys. Res. 116, C04017. (doi:1029/2010JC006687).

Hay, A.E., Zedel, L., Cheel, R., Dillon, J., 2012. On the vertical and temporal structure of flow and stress within the turbulent oscillatory boundary layer above evolving beds. Cont. Shelf Res. 46, 31-49.

Heathershaw, A.D., 1976. Measurements of turbulence in the Irish Sea benthic boundary layer. In: McCave, I.N. (Ed.), The Benthic Boundary Layer. Plenum Publishing Corporation, New York USA, ISBN: 978-1-4615-8749-1, pp. 11-31.

Holdaway, G.P., Thorne, P.D., Flatt, D., Jones, S.E., Prandle, D., 1999. Comparison between ADCP and transmissometer measurements of suspended sediment concentration. Cont. Shelf Res. 19, 421-441.

Hurther, D., Lemmin, U., Terray, E.A., 2007. Turbulent transport in the outer region of rough wall open-channel flows: the contribution of Large Coherent Shear Stress Structures (LC3S). J. Fluid Mech. 574, 465-493.

Hurther, D., Thorne, P.D., 2011. Suspension and near-bed load sediment transport processes above a migrating, sand-rippled bed under shoaling waves. J. Geophys. Res. 116, C07001. (doi:1029/2010JC006774).

Hurther, D., Thorne, P.D., Bricault, M., Lemmin, U., Baroud, J.M, 2011. A multifrequency acoustic concentration and velocity profiler (ACVP) for boundary layer measurements of fine-scale flow and sediment transport processes. Coastal Eng. 58, 294-605. (doi:10.1016/j.coastaleng.2011.01.006).

Libicki, C., Bedford, K.W., Lynch, J.F., 1989. The interpretation and evaluation of a 3-MHz acoustic backscatter device for measuring benthic boundary layer sediment dynamics. J. Acoust. Soc. Am., 1501-1511.

Moate, B.D., Thorne, P.D., 2009. Measurements and inversion of acoustic scattering from suspensions having broad size distributions. J. Acoust. Soc. Am. 126 (6), 2905. (1917).

Moate, B.D., Thorne, P.D., 2012. Interpreting acoustic backscatter from suspended sediments of different and mixed mineralogical composition. Cont. Shelf Res. $46,67-82$.

Moate, B.D., Thorne, P.D., 2013. Scattering from suspended sediments having different and mixed mineralogical compositions: comparision of laboratory measurements and theoretical predictions. J. Acoust. Soc. Am. 133 (3), 1320-1334.

Moore, S.A., Le Coz, J., Hurther, D., Paquier, A., 2012. On the application of horizontal ADCPs to suspended sediment transport surveys in rivers. Cont. Shelf Res. 46, 50-63.

Moore, S.A., Le Coz, J., Hurther, D., Paquier, A., 2013. Using multi-frequency acoustic attenuation to monitor grain size and concentration of suspended sediment in rivers. J. Acoust. Soc. Am. 133 (4), 1959-1970.

Morse, P.M., Ingard, K.U., 1987. Theoretical Acoustics. Princeton University Press p. 949. (Chap 8).

O'Hara, Murray, Hodgson, D.M., Thorne, P.D., 2012. Wave groups and the character of sediment resuspension over an evolving sandy bedforms. Cont. Shelf Res. 46 $16-30$.

Pedocchi, F., Garcia, M.H., 2012. Acoustic measurement of suspended sediment concentration profiles in an oscillatory boundary layer. Cont. Shelf Res. 46, 87-95.

Richards, S.D., Leighton, T.G., Brown, N.R., 2003. Visco-inertial absorption in dilute suspensions of irregular particles. Proc. R. Soc. London 459, 2153-2167.

Schaafsma, A.S., Hay, A.E., 1997. Attenuation in suspensions of irregularly shaped sediment particles: a two-parameter equivalent spherical scatterer model, J. Acoust. Soc. Am. 102, 1485-1502.
Sheng, J., Hay, A.E., 1988. An examination of the spherical scatterer approximation in aqueous suspensions of sand. J. Acoust. Soc. Am. 83, 598-610.

Simmons, S.M., Parsons, D.R., Best, J.L., Orfeo, O., Lane, S.N., Kostaschuk, R., Hardy, R. C., West, G., Malzone, C., Marcus, J., Pocwiardowski, P., 2010. Monitoring suspended sediment dynamics using MBES. J. Hydraul. Eng., January, 45-49.

Smerdon, A.M., Caine, S.J., 2007, A commercial multi-frequency acoustic backscatter instrument for profiling of suspended sediment size distribution and load. In: Hydraulic Measurements \& Experimental Methods Conference. Book of Extended Abstracts of the Specialty Conference. September 10-12, 2007, Lake Placid, NY, Environmental \& Water Resources Institute of the American Society of Civil Engineers and the International Association of Hydraulic Engineers.

Soulsby, R.L., 1997. Dynamics of Marine Sands. A Manual for Practical Applications. Thomas Telford publications, London, UK.

Thorne, P.D., Hardcastle, P.J., Soulsby, R.L., 1993. Analysis of acoustic measurements of suspended sediments. J. Geophys. Res. 98 (C1), 899-910.

Thorne, P.D., Hardcastle, P.J., Flatt, D., Humphery, J.D., 1994. On the use of acoustics for measuring shallow water suspended sediment processes. IEEE J. Oceanic Eng. 19 (1), 48-57.

Thorne, P.D., Holdaway, G.P, Hardcastle, P.J., 1995. Constraining acoustic backscatter estimates of suspended sediment concentration profiles using the bed echo. J. Acoust. Soc. Am. 98 (4), 2280-2288.

Thorne, P.D., Hardcastle, P.J., 1997. Acoustic measurements of suspended sediments in turbulent currents and comparison with in-situ samples. J. Acoust. Soc. Am. 101 (5), 2603-2614. (Pt. 1).

Thorne, P.D., Hanes, D.M., 2002. A review of acoustic measurement of small-scale sediment processes. Cont. Shelf Res. 22, 603-632.

Thorne, P.D., Agrawal, Y.C., Cacchione, D.A., 2007. A comparison of near-bed acoustic backscatter and laser diffraction measurements of suspended sediments. IEEE J. Ocean Eng. 32 (1), 225-235.

Thorne, P.D., Meral, R., 2008. Formulations for the scattering properties of sandy sediments for use in the application of acoustics to sediment transport. J. Cont Res. 28, 309-317.

Thorne P.D., Davies A.G., Bolanos R., 2011a. Measurement and modelling of suspended sediment size profiles above sandy rippled beds under waves. In: Published in the Proceedings of Coastal Sediments 2011, held in Miami, Florida, USA, 2 May-6 May 2011, (Eds.), Julie D Rosati, Ping Wang and Tiffany M Roberts. isbn:978-981-4355-52-0. 1620-1633.

Thorne, P.D., Hurther, D., Moate, B.D., 2011b. Acoustic inversions for measuring boundary layer suspended sediment processes. J. Acoust. Soc. Am. 130 (3) 1188-1200, http://dx.doi.org/10.1121/1.3618728.

Thorne, P.D. Hay, A.E. 2012. Introduction to thespecial issue of Continental Shelf Research; on the application of acoustics to sediment transport processes. Cont. Shelf Res. 46, 1.

Thosteson, E.D., Hanes, D.M. 1998. A Simplified method for determining sediment size and concentration from multiple frequency acoustic backscatter measurements. J. Acoust. Soc. Am. 104 (2), 820-830. (Pt.1).

Traykovski, P., 2007. Observations of wave orbital scale ripples and a nonquilibrium time-dependent model. J. Geophys. Res. 112 (C06026), 19, http://dx.doi.org/ 10.1029/2006JC003811.

van der Werf, J.J., Magar, V., Malarkey, J., Guizien, K., O’Donoghue, T., 2008. 2DV modelling of sediment transport processes over full-scale ripples in regular asymmetric oscillatory flow. Cont. Shelf Res. 28, 1040-1056.

Villard, P.V., Osborne, P.D., Vincent, C.E., 2000. Influence of wave groups on SSC patterns over vortex ripples. Cont. Shelf Res. 20 (17), 2391-2410.

Vincent, C.E., Hanes, D.M., Bowen, A.J., 1991. Acoustic measurements of suspended sand on the shoreface and the control of concentration by bed roughness. Mar Geol. 96, 1-18.

Vincent, C.E., Marsh, S.W., Webb, M.P., Osborne, P.D, 1999. Spatial and tempora structures of suspension and transport over megaripples on the shore face. J. Geophys. Res. 104 (C5), 11,215-11,224.

Vogt, R.H., Neubauer, W.G., 1976. Relationship between acoustic reflection and vibrational modes of elastic spheres. J. Acoust. Soc. Am. 60 (1), 15-22.

Wilhelmij, P, Denbigh, P., 1984. A statistical approach to determining the number density of random scatterers from backscattered pulses. J. Acoust. Soc. Am. 76 (6), 1810-1818. 